\title{
New Numerical Solution of von Karman Equation of Lengthwise Rolling
}

\author{
Rudolf Pernis ${ }^{1}$ and Tibor Kvackaj ${ }^{2}$ \\ ${ }^{1}$ Alexander Dubček University of Trenčín, Pri Parku 19, 91106 Trenčín-Záblatie, Slovakia \\ ${ }^{2}$ Technical University of Košice, Faculty of Metallurgy, 04200 Košice, Slovakia
}

Correspondence should be addressed to Tibor Kvackaj; tibor.kvackaj@tuke.sk

Received 10 June 2015; Accepted 16 August 2015

Academic Editor: Sutasn Thipprakmas

Copyright ( 2015 R. Pernis and T. Kvackaj. This is an open access article distributed under the Creative Commons Attribution License, which permits unrestricted use, distribution, and reproduction in any medium, provided the original work is properly cited.

The calculation of average material contact pressure to rolls base on mathematical theory of rolling process given by Karman equation was solved by many authors. The solutions reported by authors are used simplifications for solution of Karman equation. The simplifications are based on two cases for approximation of the circular arch: (a) by polygonal curve and (b) by parabola. The contribution of the present paper for solution of two-dimensional differential equation of rolling is based on description of the circular arch by equation of a circle. The new term relative stress as nondimensional variable was defined. The result from derived mathematical models can be calculated following variables: normal contact stress distribution, front and back tensions, angle of neutral point, coefficient of the arm of rolling force, rolling force, and rolling torque during rolling process. Laboratory cold rolled experiment of $\mathrm{CuZn} 30$ brass material was performed. Work hardening during brass processing was calculated. Comparison of theoretical values of normal contact stress with values of normal contact stress obtained from cold rolling experiment was performed. The calculations were not concluded with roll flattening.

\section{Introduction}

It is likely that the author Karman [1] in the year 1925 was the first to submit equation of power balance in the roll gap and derived the two-dimensional differential equation which described lengthwise rolling process. The following simplifications were received:

(i) Rolled material has rectangular cross section with initial thickness $h_{0}$ and is deformed by cylindrical rolls to final thickness $h_{1}$ without any action of front and back tensions.

(ii) Spread is negligible.

(iii) Friction coefficient between rolls and rolled material is constant.

(iv) Rolls are rigid without any elastic deformation and bending.

(v) Rolled material is homogenous and without any elastic deformation. (vi) Tresca condition of plasticity: $\sigma_{1}-\sigma_{3}=\sigma_{a}$.

(vii) Flow stress of material in rolling gap is constant.

(viii) Rolling speed is constant.

Geometrical description of roll gap is given in Figure 1 and essential formulas are presented in the appendix. From graphical scheme of roll gap the result is that all forces have to be at balance which was the base for derivation two-dimensional differential equation describing lengthwise rolling process. The procedure of derivation of the twodimensional differential equation can be found in the classical literature of rolling, for example, Avitzur [2], Hensel and Spittel [3], and Mielnik [4]. More recent literatures are the publications Hajduk and Konvičný [5] and Kollerová et al. [6]. The stress state in rolling gap describes differential equation:

$$
\frac{d \sigma_{x}}{d x}-\frac{\sigma_{n}-\sigma_{x}}{y} \cdot \frac{d y}{d x} \pm \frac{\tau}{y}=0
$$




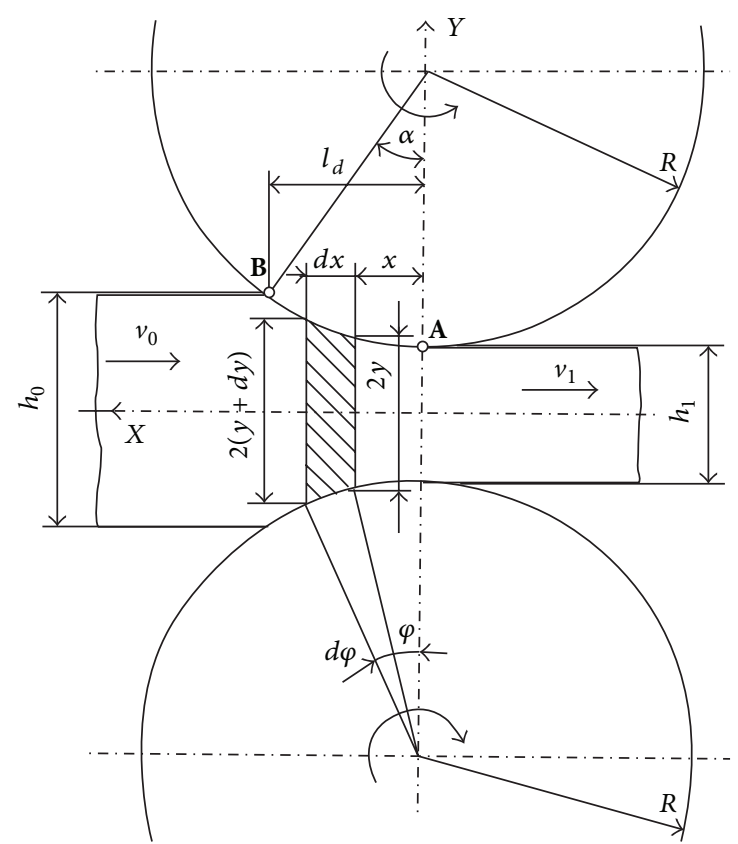

FIGURE 1: Determination of geometric relationships.

where plus sign $(+)$ is for forward slip zone, minus sign (-) is for backward slip zone, $\sigma_{n}$ is normal contact stress on rolls, $\tau$ is the shear stress between rolls and rolling material, and $x, y$ are the coordinates of the cylinder touching the rolled material.

The Tresca condition of plasticity is used for solution of (1):

$$
-\sigma_{x}-\left(-\sigma_{n}\right)=\sigma_{n}-\sigma_{x}=\sigma_{a}
$$

where $\sigma_{y} \doteq \sigma_{n}$ is the maximal principal stress (vertical direction), $\sigma_{x}$ is the minimal principal stress (horizontal direction), $\sigma_{a}$ is the flow stress [7-9], and $\tau$ is the shear stress that is proportional to normal contact stress $\sigma_{n}$ according to formula:

$$
\tau=f \cdot \sigma_{n}
$$

where $f$ is the friction coefficient between rolls and rolled material.

Substituting (2) into (3) the following formula will be obtained with only one stress variable $d \sigma_{n}$ :

$$
\frac{d \sigma_{n}}{d x}-\frac{\sigma_{a}}{y} \cdot \frac{d y}{d x} \pm \frac{f}{y} \cdot \sigma_{n}=0
$$

Flow stress and friction coefficient are as a constant in (4). If differential equation (4) is divided by flow stress $\sigma_{a}$ it is possible to obtain relative normal contact stress $\bar{\sigma}_{n}$ :

$$
\bar{\sigma}_{n}=\frac{\sigma_{n}}{\sigma_{a}}
$$

where $\bar{\sigma}$ is a function.
Substituting (5) into (4) the following form is obtained:

$$
\frac{d \bar{\sigma}_{n}}{d x}-\frac{1}{y} \cdot \frac{d y}{d x} \pm \frac{f}{y} \cdot \bar{\sigma}_{n}=0
$$

The application equation (5) can be excluded from (4) material constant (flow stress $\sigma_{a}$ ). Thus (6) become independent from the rolled material. This differential equation is a linear differential equation of first order with the right side and has a general transcription: $\bar{\sigma}_{n}=\bar{\sigma}_{n}(x, y)$. The coordinates $x$ and $y$ are defining the geometry of the cylinder which is connected to the rolling material. The variable $y$ is a function of the $x$ coordinate. If equation of a circle is substituting into (6), so differential equation is obtained which has no analytical solution till now. The author Orowan [10] presented the solution of differential equation (1) based on analyticalgraphical principle. He defined procedure for calculation rolling force, rolling torque, and electric input for hot and cold rolling processes. In the literature [11] showed solution of differential equation (1) by simulation on analog computer. The authors of [12] solved this case with the use of the circular arc but the result is purely numerical solution. The author of [13] presented simplified analytical solution with acceptance of material work hardening during cold rolling process. The development in computational software allowed to solve this task by using finite element method as was given in the literature [14].

\section{Experimental Material and Methods}

The investigated material was annealed brass CuZn30 with chemical composition corresponding to standard DIN 17660, W.No. 2.0265. The sample sizes before rolling were $h \times b \times l$ $=3,4 \times 30 \times 150 \mathrm{~mm}$. The samples were laboratory rolled using duo mill (the diameter of rolls $D=210 \mathrm{~mm}$ ) with constant circumferential speed of rolls $v_{\text {roll }}=0,66 \mathrm{~m} / \mathrm{s}$ at ambient temperatures. The temperature of a sample surface immediately after rolling was measured using a thermovision camera and was from interval $T \in\langle 20 ; 40\rangle\left[{ }^{\circ} \mathrm{C}\right]$. The cold rolling reductions of thickness were made in an interval $\varepsilon_{\text {cold }} \in\langle 0 ; 30\rangle[\%]$ as one pass deformation. Deformation forces during samples processing by cold rolling were measured by tensometric elements with signal registration in Spider apparatus. The static tensile test was performed in accordance with STN EN 10 002-1 standard.

The differential equation of lengthwise rolling process given by formula (1) has no analytical solution when contact arc is described with the equation of the circle. For solution of this problem a new combined analytical and numerical approach is presented.

\section{Analysis and Results}

3.1. Analysis of the Present Solution of Differential Equation Describing Stress Condition in Rolling Gap. The present solutions of differential equation of rolling were based on the same simplifications because the form of equation is 


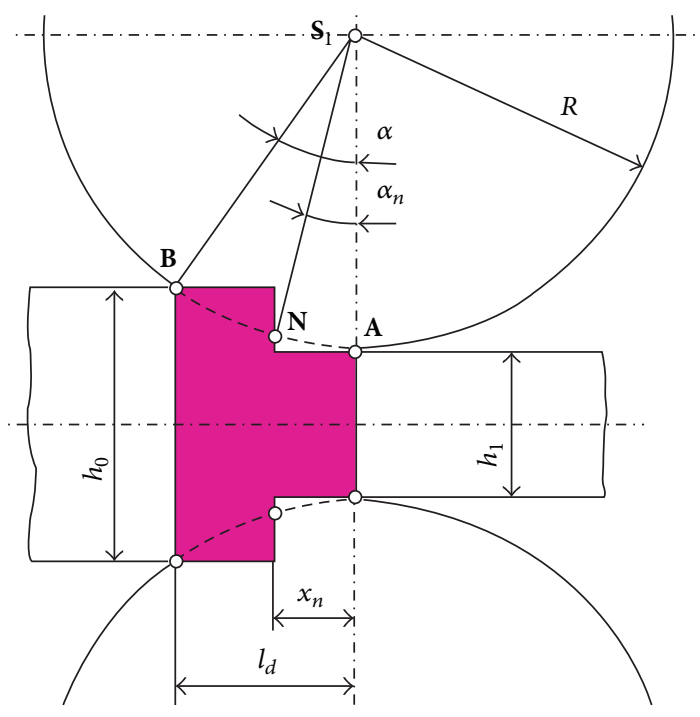

FIgURE 2: The contact arc according to Korolev.

complicated. More than twenty solutions of differential equation are described in the literature. The present solutions of differential equation (1) can be divided into following groups:

(i) Geometry of cylinder: the circular arc of the cylinder is being approximated by simplifying curves as straight line, polygonal line, and parabola or in some cases using equation of a circle.

(ii) Friction conditions between rolls and rolled material:

(a) The shear stress between rolls and rolling material is constant: $\tau=f \cdot \sigma_{a}$.

(b) The shear stress between rolls and rolling material is changing: $\tau=f \cdot \sigma_{n}$.

(c) Friction coefficient is $f=0$.

(iii) Mathematical methods of solution: analytical, analytical-graphical, analytical-numerical, numerical, and semiempirical.

(iv) Work hardening of rolled material: most of the solution is based on assumption that material during processing is without work hardening. In a few isolated cases solution with work hardening of rolled material was used.

In the following part most frequently ways for calculation of $\bar{\sigma}$ function which are fall to first group will be analyzed.

3.1.1. The Solution according to Korolev. The author Korolev [15] presented solution based on approximation of circular arch by parallel step function which is shown in Figure 2. The circular arch (BA) is approximated by polygonal line. Neutral point $\mathbf{N}$ divides space of rolling gap on parts: backward slip zone (BN) and forward slip zone (NA). Position of neutral point is determined by coordinate $x_{n}$ :

$$
\frac{x_{n}}{l_{d}}=\frac{h_{1}}{h_{0}+h_{1}} \text {. }
$$

The distribution functions describing relative normal contact stress in rolling gap were divided separately for backward and forward slip zone.

The distribution function describing relative normal contact stress $\bar{\sigma}_{n B}$ in backward slip zone of rolling gap is as follows:

$$
\bar{\sigma}_{n B}=e^{m_{0}\left(1-x / l_{d}\right)},
$$

where

$$
m_{0}=2 f \cdot \frac{l_{d}}{h_{0}} .
$$

The distribution function describing relative normal contact stress $\bar{\sigma}_{n F}$ in forward slip zone of rolling gap is as follows:

$$
\bar{\sigma}_{n F}=e^{m_{1} \cdot\left(x / l_{d}\right)}
$$

where

$$
m_{1}=2 f \cdot \frac{l_{d}}{h_{1}} .
$$

The author of [15] on the basis of the previous distribution function derived an equation for calculation $\bar{\sigma}$ function as follows:

$$
\bar{\sigma}_{\text {Kor }}=\frac{1}{m_{\text {Kor }}}\left(e^{m_{\text {Kor }}}-1\right)
$$

where $m_{\text {Kor }}$ is constant:

$$
m_{\mathrm{Kor}}=f \cdot \frac{l_{d}}{h_{\mathrm{av}}} .
$$

3.1.2. The Solution according to Tselikov. The authors Tselikov et al. [16] presented solution based on approximation of circular arch by straight line function which is shown in Figure 3. The circular arch (BA) is approximated by straight line. Neutral point $\mathbf{N}$ divides space of rolling gap on parts: backward slip zone (BN) and forward slip zone (NA). Position of neutral point is determined by coordinate $x_{n}$ and thickness in neutral section $h_{n}$ was derived as follows:

$$
\frac{h_{n}}{h_{1}}=\left[\frac{1}{m+1} \cdot\left(1+\sqrt{1+\left(m^{2}-1\right) \cdot\left(\frac{h_{0}}{h_{1}}\right)^{m}}\right)\right]^{1 / m},
$$

where $m$ is constant:

$$
m \equiv m_{\mathrm{Tsel}}=\frac{2 f \cdot l_{d}}{h_{0}-h_{1}} .
$$

The distribution functions describing relative normal contact stress $\bar{\sigma}_{n F}$ in forward slip zone and $\bar{\sigma}_{n B}$ in backward slip zone of rolling gap are as follows:

$$
\begin{aligned}
& \bar{\sigma}_{n B}=\frac{1}{m}\left[(m-1) \cdot\left(\frac{h_{0}}{2 y}\right)^{m}+1\right], \\
& \bar{\sigma}_{n F}=\frac{1}{m}\left[(m+1) \cdot\left(\frac{2 y}{h_{1}}\right)^{m}-1\right] .
\end{aligned}
$$




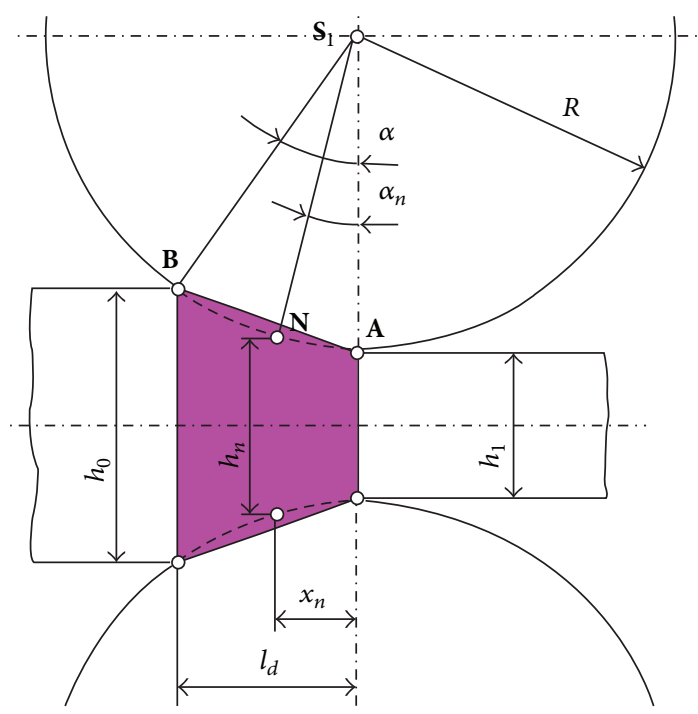

FIgURE 3: The contact arc according to Tselikov.

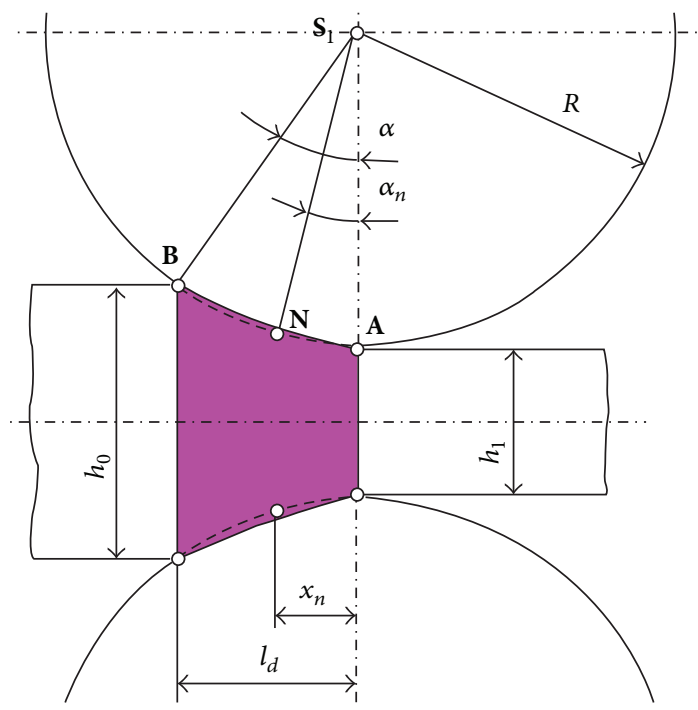

Figure 4: The contact arc approximated by a parabola.

The authors of [16] on the basis of the previous distribution functions derived an equation for calculation $\bar{\sigma}$ function as follows:

$$
\bar{\sigma}_{\text {Tsel }}=\frac{2(1-\varepsilon)}{\varepsilon(m-1)} \cdot \frac{h_{n}}{h_{1}} \cdot\left[\left(\frac{h_{n}}{h_{1}}\right)^{m}-1\right] .
$$

From (17) the result is that, for condition $m=1, \bar{\sigma}$ function is discontinuous.

3.1.3. The Solution according to Bland and Ford. The authors Bland and Ford [17] presented solution based on approximation of circular arch by parabolic function which is shown in Figure 4. The circular arch (BA) is approximated by part of parabola. Neutral point $\mathbf{N}$ divides space of rolling gap on parts: backward slip zone (BN) and forward slip zone
(NA). The authors defined neutral angle $\alpha_{n}$ according to the following formula:

$$
\begin{aligned}
\alpha_{n} & \sqrt{\frac{h_{1}}{R}} \\
& \cdot \operatorname{tg}\left[\frac{1}{2} \cdot \operatorname{arctg}\left(\sqrt{\frac{R}{h_{1}}} \cdot \alpha\right)-\frac{1}{4 f} \cdot \sqrt{\frac{h_{1}}{R}} \cdot \ln \frac{h_{0}}{h_{1}}\right] .
\end{aligned}
$$

The distribution functions describing relative normal contact stress $\bar{\sigma}_{n F}$ in forward slip zone and $\bar{\sigma}_{n B}$ in backward slip zone of rolling gap are as follows:

$$
\begin{aligned}
& \bar{\sigma}_{n B}=\frac{h_{1}+R \varphi^{2}}{h_{0}} \cdot e^{f\left(H_{0}^{*}-H^{*}\right)}, \\
& \bar{\sigma}_{n F}=\frac{h_{1}+R \varphi^{2}}{h_{1}} \cdot e^{f \cdot H^{*}},
\end{aligned}
$$

where

$$
\begin{aligned}
& H^{*}=2 \sqrt{\frac{R}{h_{1}}} \operatorname{arctg}\left(\sqrt{\frac{R}{h_{1}}} \cdot \varphi\right), \\
& H_{0}^{\cdot}=2 \sqrt{\frac{R}{h_{1}}} \operatorname{arctg}\left(\sqrt{\frac{R}{h_{1}}} \cdot \alpha\right) .
\end{aligned}
$$

The authors of [17] on the basis of the previous distribution functions derived an equation for calculation $\bar{\sigma}$ function as follows:

$$
\begin{gathered}
\bar{\sigma}_{\text {Bland-Ford }}=\sqrt{\frac{1-\varepsilon}{\varepsilon} m}\left[(1-\varepsilon) \cdot e^{2 m \cdot \operatorname{arctg}(\sqrt{\varepsilon /(1-\varepsilon)})}\right. \\
\cdot \int_{t_{n}}^{t_{\alpha}}\left(1+m^{2} t^{2}\right) \cdot e^{-2 m \cdot \operatorname{arctg}(m t)} \cdot d t \\
\left.+\int_{0}^{t_{n}}\left(1+m^{2} t^{2}\right) \cdot e^{2 m \cdot \operatorname{arctg}(m t)} \cdot d t\right]
\end{gathered}
$$

where $m$ is constant and $t_{n}, t_{\alpha}$ are limits of integration, $t=$ $\varphi / f$ :

$$
\begin{aligned}
& m \equiv m_{\text {Bland-Ford }}=f \cdot \sqrt{\frac{R}{h_{1}}}, \\
& t_{n}=\frac{\alpha_{n}}{f}, \\
& t_{\alpha}=\frac{1}{m} \cdot \sqrt{\frac{\varepsilon}{1-\varepsilon}} .
\end{aligned}
$$

3.1.4. The Solution according to Sims. The author Sims [18] presented solution of differential equation without consideration of friction coefficient. Roll radius is considered with 
elastic flattening $R=R^{\prime}$. The ratio $h_{n} / h_{1}$ was derived from the following formula:

$$
\frac{h_{n}}{h_{1}}=1+\frac{2 R}{h_{1}}\left(1-\cos \alpha_{n}\right) \text {, }
$$

where

$$
\begin{aligned}
\alpha_{n} & =\sqrt{\frac{h_{1}}{R}} \cdot \operatorname{tg}\left(\frac{\pi}{16} \cdot \sqrt{\frac{h_{1}}{R}} \cdot \ln (1-\varepsilon)\right. \\
& \left.+\frac{1}{2} \operatorname{arctg}\left(\sqrt{\frac{R}{h_{1}}} \cdot \alpha\right)\right) .
\end{aligned}
$$

The distribution functions describing relative normal contact stress $\bar{\sigma}_{n F}$ in forward slip zone and $\bar{\sigma}_{n B}$ in backward slip zone of rolling gap were calculated according to the following formulae:

$$
\begin{aligned}
& \bar{\sigma}_{n B}=1+\frac{\pi}{4} \ln \left((1-\varepsilon)\left(1+\frac{R}{h_{1}} \varphi^{2}\right)\right) \\
& +2 \sqrt{\frac{R}{h_{1}}}\left(\operatorname{arctg}\left(\sqrt{\frac{R}{h_{1}}} \cdot \alpha\right)-\operatorname{arctg}\left(\sqrt{\frac{R}{h_{1}}} \cdot \varphi\right)\right), \\
& \bar{\sigma}_{n F}=1+\frac{\pi}{4} \ln \left(1+\frac{R}{h_{1}} \varphi^{2}\right)+2 \sqrt{\frac{R}{h_{1}}} \\
& \cdot \operatorname{arctg}\left(\sqrt{\frac{R}{h_{1}}} \cdot \varphi\right) .
\end{aligned}
$$

The author of [18] on the basis of the previous distribution functions derived an equation for calculation $\bar{\sigma}$ function as follows:

$$
\begin{aligned}
\bar{\sigma}_{\text {Sim }}= & \frac{\pi}{2} \sqrt{\frac{1-\varepsilon}{\varepsilon}} \operatorname{arctg}\left(\sqrt{\frac{\varepsilon}{1-\varepsilon}}\right)-\frac{\pi}{4}-\sqrt{\frac{1-\varepsilon}{\varepsilon}} \\
& \cdot \sqrt{\frac{R}{h_{1}}} \cdot \ln \frac{h_{n}}{h_{1}}+\frac{1}{2} \sqrt{\frac{1-\varepsilon}{\varepsilon}} \cdot \sqrt{\frac{R}{h_{1}}} \cdot \ln \frac{1}{1-\varepsilon} .
\end{aligned}
$$

3.1.5. The Modification Smiryagin Solution by Pernis. The authors of [19] for solution of (1) presented modification of Tselikov solution using a parabolic approximation function of the circular arc as is shown in Figure 4. The distribution functions describing relative normal contact stress $\bar{\sigma}_{n F}$ in forward slip zone and $\bar{\sigma}_{n B}$ in backward slip zone of rolling gap were calculated according to the following formulae:

$$
\begin{aligned}
& \bar{\sigma}_{n B}=\left[1-\left(\frac{u_{0}}{m}-\frac{1}{m^{2}}\right)\right] e^{m\left(u_{0}-u\right)}+2\left(\frac{u}{m}-\frac{1}{m^{2}}\right), \\
& \bar{\sigma}_{n F}=\left(1+\frac{1}{m^{2}}\right) e^{m u}-2\left(\frac{u}{m}+\frac{1}{m^{2}}\right),
\end{aligned}
$$

where $m, u_{0}$ are constants:

$$
\begin{aligned}
& m \equiv m_{\text {Smir-Per }}=\frac{2 f \cdot l_{d}}{\sqrt{h_{1} \cdot\left(h_{0}-h_{1}\right)}}, \\
& u_{0}=\operatorname{arctg}\left(\sqrt{\frac{\varepsilon}{1-\varepsilon}}\right) .
\end{aligned}
$$

At this point the Tselikov solution of differential equation (1) was completed. The function for calculation of neutral angle and average relative normal contact stress has not been determined. Additional calculations were carried out by the author of [20] which are derived by additional equations describing neutral angle and average relative normal contact stress. Location of the neutral point was determined through using the value $u_{n}$ (dimensionless number) and is expressed by an explicit equation:

$$
\begin{aligned}
& \left(0,5 m^{2}-m \cdot u_{0}+1\right) \cdot e^{m\left(u_{0}-u_{n}\right)}-\left(0,5 m^{2}+1\right) \cdot e^{m \cdot u_{n}} \\
& \quad+2 m \cdot u_{n}=0 .
\end{aligned}
$$

The neutral point can be obtained from the following formula:

$$
\sin \alpha_{n}=\sqrt{\frac{1-\varepsilon}{\varepsilon}} \cdot \operatorname{tg} u_{n} \cdot \sin \alpha .
$$

The authors of [20] on the basis of the previous distribution functions derived an equation for calculation $\bar{\sigma}$ function as follows:

$$
\begin{aligned}
& \bar{\sigma}_{\text {Smir-Per }}=\frac{2}{m \cdot u_{0}} \cdot\left[\left(1+\frac{2}{m^{2}}\right) \cdot\left(e^{m \cdot u_{n}}-1\right)+\frac{u_{0}^{2}}{2}\right. \\
&\left.-u_{n} \cdot\left(u_{n}+\frac{2}{m}\right)\right] .
\end{aligned}
$$

\subsection{New Approach for Solution of von Karman Differential} Equation. The following approach represents analytic and numerical solution of (1) where for description of the circular arc equation of circle is applied as is shown in Figure 5. Equation (6) can be classified as the first-order linear differential equation with right side:

$$
\frac{d \bar{\sigma}_{n}}{d x} \pm \frac{f}{y} \bar{\sigma}_{n}=\frac{1}{y} \cdot \frac{d y}{d x} .
$$

Many authors [21-23] have dealt with the solution of this type of differential equation. Firstly (35) will be solved as equation without right side:

$$
\frac{d \bar{\sigma}_{n}}{d x} \pm \frac{f}{y} \bar{\sigma}_{n}=0
$$

which can be divided into the two following differential equations:

$$
\begin{aligned}
& \frac{d \bar{\sigma}_{n B}}{d x}+\frac{f}{y} \bar{\sigma}_{n B}=0, \\
& \frac{d \bar{\sigma}_{n F}}{d x}-\frac{f}{y} \bar{\sigma}_{n F}=0 .
\end{aligned}
$$




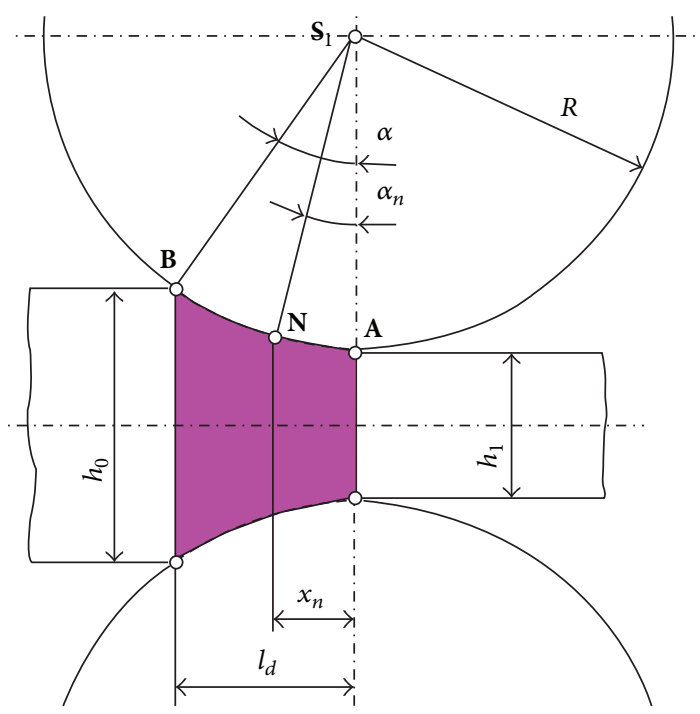

FIgURE 5: The contact arc described by equation of circle.

The following solution of (37) is valid for backward slip zone:

$$
\int \frac{d \bar{\sigma}_{n B}}{\bar{\sigma}_{n B}}=-f \cdot \int \frac{d x}{y} .
$$

The calculation of integral of the left side of the equation can be carried out as follows:

$$
\ln \left(\bar{\sigma}_{n B}\right)=-f \cdot \int \frac{d x}{y}, \quad \bar{\sigma}_{n B}=c \cdot e^{-f \cdot \int(d x / y)} .
$$

If the integral is the exponent and also is a known function $F_{1}(x)$ so it is possible to write

$$
\begin{aligned}
F_{1}(x) & =\int \frac{d x}{y}, \\
\text { respectively } \frac{d F_{1}(x)}{d x} & =\frac{1}{y} .
\end{aligned}
$$

Equation (40) is representing general form of (37) and $c$ is function of variable $x$ :

$$
\bar{\sigma}_{n}=c(x) \cdot e^{-f \cdot F_{1}(x)} \quad(c=c(x)) .
$$

Differentiating the equation is obtained from

$$
\frac{d \bar{\sigma}_{n}}{d x}=\frac{d c(x)}{d x} \cdot e^{-f \cdot F_{1}(x)}-c(x) \cdot \frac{d F_{1}(x)}{d x} \cdot e^{-f \cdot F_{1}(x)} .
$$

Equations (42) and (43) are substituting into (35):

$$
\begin{gathered}
\frac{d c(x)}{d x} \cdot e^{-f \cdot F_{1}(x)}-c(x) \cdot \frac{F_{1}(x)}{d x} \cdot e^{-f \cdot F_{1}(x)}+c(x) \\
\cdot \frac{F_{1}(x)}{d x} \cdot e^{-f \cdot F_{1}(x)}=\frac{1}{y} \cdot \frac{d y}{d x}
\end{gathered}
$$

from which function $c(x)$ can be determined as follows:

$$
\begin{aligned}
\frac{d c(x)}{d x} \cdot e^{-f \cdot F_{1}(x)} & =\frac{1}{y} \cdot \frac{d y}{d x} \\
c(x) & =\int e^{f \cdot F_{1}(x)} \cdot \frac{d y}{y}+C,
\end{aligned}
$$

where $C$ is an integration constant that must be determined from the boundary conditions for each segment separately (backward and forward slip zone).

Substituting (45) into (42) will obtain the general form of (35) for backward slip zone:

$$
\bar{\sigma}_{n B}=e^{-f \cdot F_{1}(x)} \cdot\left(C_{B}+\int e^{f \cdot F_{1}(x)} \cdot \frac{d y}{y}\right) .
$$

Determination of ratio $d y / y$ and function $F_{1}(x)$ can be done using the equation of the circle:

$$
\begin{aligned}
x^{2}+\left(y-y_{0}\right)^{2} & =R^{2}, \\
y_{0} & =R+\frac{h_{1}}{2} .
\end{aligned}
$$

Separating the variable $y$ from (47) and its differentiation is obtained:

$$
\begin{aligned}
y & =R+\frac{h_{1}}{2} \pm \sqrt{R^{2}-x^{2}}, \\
d y & =\frac{x \cdot d x}{\sqrt{R^{2}-x^{2}}} .
\end{aligned}
$$

The next solution is apparent from the description:

$$
\begin{aligned}
\frac{d y}{y} & =\frac{x / \sqrt{R^{2}-x^{2}}}{R+h_{1} / 2-\sqrt{R^{2}-x^{2}}} d x \\
& =\frac{x / R}{\sqrt{1-(x / R)^{2}}} \cdot \frac{d x}{R\left(m-\sqrt{1-(x / R)^{2}}\right)},
\end{aligned}
$$

where the constant values are included in one constant $m$ :

$$
m=1+\frac{h_{1}}{2 R} .
$$

The simplification of (50) can be obtained with transformation from Cartesian coordinates to polar coordinates with use of the following substitution with respect to Figure 6:

$$
\frac{x}{R}=\sin \varphi,
$$

$$
\text { respectively } d x=R \cos \varphi \cdot d \varphi \text {. }
$$

Substituting (52) into (50) will obtain the form

$$
\begin{aligned}
\frac{d y}{y} & =\frac{\sin \varphi}{\sqrt{1-\sin ^{2} \varphi}} \cdot \frac{R \cos \varphi \cdot d \varphi}{R\left(m-\sqrt{1-\sin ^{2} \varphi}\right)} \\
& =\frac{\sin \varphi}{m-\cos \varphi} \cdot d \varphi=F_{2}(\varphi) \cdot d \varphi,
\end{aligned}
$$

where a new function $F_{2}(\varphi)$ is expressed as follows:

$$
F_{2}(\varphi)=\frac{\sin \varphi}{m-\cos \varphi} .
$$




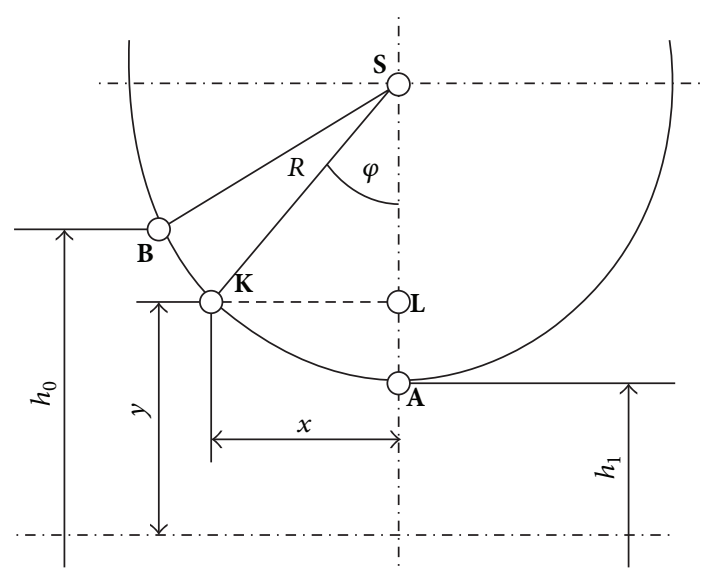

FIGURE 6: Definition of the point $\mathbf{K}[x ; y]$.

The function $F_{1}(\varphi)$ can be expressed from (41) according to the solution defined in work [24] as follows:

$$
\begin{aligned}
F_{1}(x) & =\int \frac{d x}{y}=\int \frac{d x}{y_{0}-\sqrt{R^{2}-x^{2}}} \\
& =\int \frac{d x / R}{m-\sqrt{1-(x / R)^{2}}} .
\end{aligned}
$$

Transformation of function $F_{1}(x)$ from Cartesian coordinates to function $F_{1}(\varphi)$ in polar coordinates will be obtained:

$$
F_{1}(\varphi)=\int \frac{\cos \varphi \cdot d \varphi}{m-\sqrt{1-\sin ^{2} \varphi}}=\int \frac{\cos \varphi}{m-\cos \varphi} \cdot d \varphi,
$$

where

$$
I_{1}=\int \frac{\cos \varphi}{m-\cos \varphi} \cdot d \varphi
$$

using the following substitutions:

$$
\begin{aligned}
\operatorname{tg} \frac{\varphi}{2} & =z, \\
\cos \varphi & =\frac{1-z^{2}}{1+z^{2}}, \\
d \varphi & =\frac{2 d z}{1+z^{2}}
\end{aligned}
$$

which are inserted into (57) and the integral is obtained:

$$
I_{1}=\frac{2}{m+1} \int \frac{1-z^{2}}{\left(1+z^{2}\right) \cdot\left(a+z^{2}\right)} \cdot d z
$$

where

$$
a=\frac{m-1}{m+1} .
$$

By decomposition of the integral of (59) to partial fractions the following formula is obtained:

$$
I_{1}=\frac{2}{m+1}\left(\frac{2}{a-1} \int \frac{d z}{1+z^{2}}+\frac{1+a}{1-a} \int \frac{d z}{a+z^{2}}\right),
$$

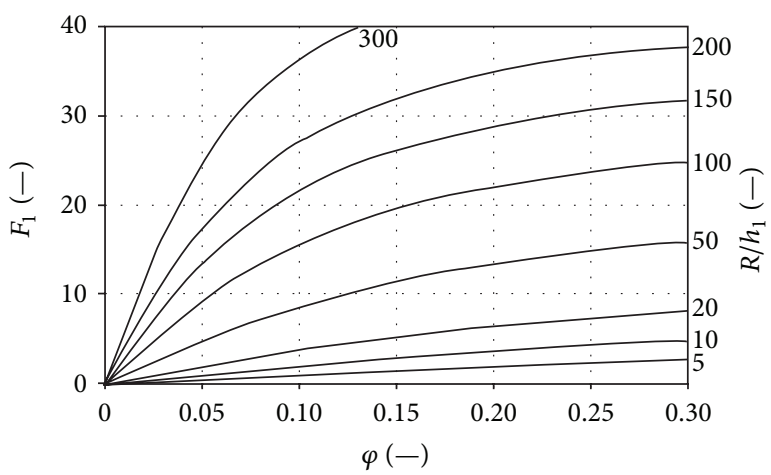

FIgURE 7: Graphical visualization of function $F_{1}(\varphi)$.

and calculation of subintegrals in (61) enters to the form

$$
I_{1}=\frac{2}{m+1}\left(\frac{2}{a-1} \operatorname{arctg} z+\frac{1+a}{1-a} \cdot \frac{1}{\sqrt{a}} \operatorname{arctg} \frac{z}{\sqrt{a}}\right) .
$$

Substituting (60) into (62) will obtain the form

$$
I_{1}=-2 \operatorname{arctg} z+\frac{2 m}{\sqrt{m^{2}-1}} \operatorname{arctg}\left(\sqrt{\frac{m+1}{m-1}} \cdot z\right)
$$

and also substituting (58) into (63) integral $I_{1}$ will be in the form

$$
I_{1}=\frac{2 m}{\sqrt{m^{2}-1}} \operatorname{arctg}\left(\sqrt{\frac{m+1}{m-1}} \cdot \operatorname{tg} \frac{\varphi}{2}\right)-\varphi .
$$

Integral $I_{1}$ represents the analytical form of the function $F_{1}(\varphi)$ :

$$
F_{1}(\varphi)=\frac{2 m}{\sqrt{m^{2}-1}} \cdot \operatorname{arctg}\left(\sqrt{\frac{m+1}{m-1}} \cdot \operatorname{tg} \frac{\varphi}{2}\right)-\varphi .
$$

Transformation Cartesian coordinate $x$ to polar coordinate $\varphi$ in (46) and substituting function $F_{2}(\varphi)$ are obtained from the form

$$
\bar{\sigma}_{n B}=e^{-f \cdot F_{1}(\varphi)} \cdot\left(C_{B}+\int e^{f \cdot F_{1}(\varphi)} \cdot F_{2}(\varphi) \cdot d \varphi\right) .
$$

Limits of integration in (66) are $\langle\varphi ; \alpha\rangle$. Solution of (38) describing the forward slip process can be obtained by the analogical way as reported for solving (37). The final solution of (38) of relative normal contact stress in forward slip zone will be as follows:

$$
\bar{\sigma}_{n F}=e^{f \cdot F_{1}(\varphi)} \cdot\left(C_{F}+\int e^{-f \cdot F_{1}(\varphi)} \cdot F_{2}(\varphi) \cdot d \varphi\right) .
$$

Limits of integration are $\langle\varphi ; \alpha\rangle$. From (66) and (67) the result is that the relative normal contact stress is proportional to the product of two functions: $\bar{\sigma}_{n} \approx e^{f \cdot F_{1}} \cdot F_{2}$.

Graphical visualization of function $F_{1}(\varphi)$ depending on angle coordinate and ratio $R / h_{1}$ is given in Figure 7. Functions $F_{1}(\varphi)$ throughout the observed interval are monotonically increasing. With increasing of ratio $R / h_{1}$ also functions 


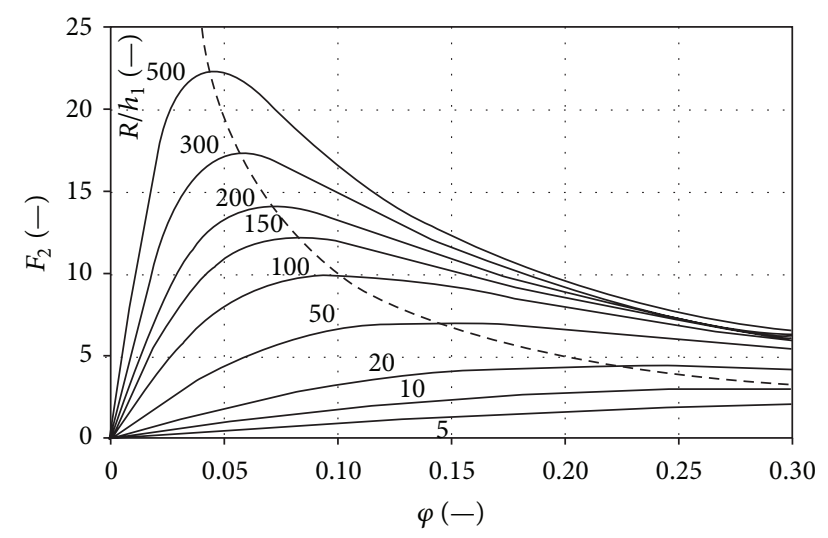

FIGURE 8: Graphical visualization of function $F_{2}(\varphi)$.

$F_{1}(\varphi)$ are rising. The function $F_{1}(\varphi)$ during rolling process represents wrapping angle of roll $\beta$ by rolled material $(\beta$ is not gripping angle). Graphical visualization of function $F_{2}(\varphi)$ depending on angle coordinate is given in Figure 8. Local maximum of functions $F_{2}(\varphi)$ increases with increasing ratio $R / h_{1}$. Location point expressing the maximum value of the function $F_{2}(\varphi)$ is determined from the condition $d F_{2}(\varphi) / d \varphi=0$ :

$$
\cos \varphi_{0}=\frac{1}{m}
$$

where $\varphi_{0}$ is coordinate in maximum and $m$ is constant of differential equation given by (51).

Maximal value of function $F_{2}(\varphi)$ can be determined from (54) for coordinate $\varphi_{0}$ as follows:

$$
F_{2, \text { max }}\left(\varphi_{0}\right)=\frac{1}{\sqrt{m^{2}-1}} .
$$

The broken line in Figure 8 shows the positions of the maximal values. The integration constants in (66) and (67) must be determined from Tresca condition of plasticity. Stress state according to Figure 1 can be described for point $\mathbf{A}$ (output of material from rolling gap) and for point $\mathbf{B}$ (input of material to rolling gap) using the following formulae:

$$
\begin{aligned}
& 0-\left(-\sigma_{n B}\right)=\sigma_{n B}=\sigma_{a}, \\
& 0-\left(-\sigma_{n F}\right)=\sigma_{n F}=\sigma_{a} .
\end{aligned}
$$

Equations (70) are normalized according to flow stress:

$$
\begin{aligned}
\frac{\sigma_{n B}}{\sigma_{a}} & =\frac{\sigma_{a}}{\sigma_{a}} \Longrightarrow \bar{\sigma}_{n B}=1 \quad(\varphi=\alpha), \\
\frac{\sigma_{n F}}{\sigma_{a}} & =\frac{\sigma_{a}}{\sigma_{a}} \Longrightarrow \bar{\sigma}_{n F}=1 \quad(\varphi=0) .
\end{aligned}
$$

The determination of integration constants is performed with assumption without work hardening of material during rolling process, without forward and backward stretching, and without roll flattening. In the input and output plane of rolling material the stress is $\sigma_{x}=0$. The integration constant
$C_{B}$ for the backward slip zone $(\varphi=\alpha)$ is found from (66) as follows:

$$
C_{B}=e^{f \cdot F_{1}(\alpha)},
$$

where $F_{1}(\alpha)$ is a known function given by (65). The integration constant $C_{F}$ for the forward slip zone $(\varphi=0)$ is found from (67) as follows:

$$
C_{F}=1 \text {. }
$$

The total form of equation for the calculation of the relative normal contact stress $\bar{\sigma}_{n B}$ for the backward slip zone can be written as follows:

$$
\begin{gathered}
\bar{\sigma}_{n B}=e^{-f \cdot\left[\left(2 m / \sqrt{m^{2}-1}\right) \cdot \operatorname{arctg}(\sqrt{(m+1) /(m-1)} \cdot \operatorname{tg}(\varphi / 2))-\varphi\right]} \\
\cdot\left(e^{f \cdot\left[\left(2 m / \sqrt{m^{2}-1}\right) \cdot \operatorname{arctg}(\sqrt{(m+1) /(m-1)} \cdot \operatorname{tg}(\alpha / 2))-\alpha\right]}\right. \\
+\int_{\varphi}^{\alpha} e^{f \cdot\left[\left(2 m / \sqrt{m^{2}-1}\right) \cdot \operatorname{arctg}(\sqrt{(m+1) /(m-1)} \cdot \operatorname{tg}(\varphi / 2))-\varphi\right]} \\
\left.\cdot \frac{\sin \varphi}{m-\cos \varphi} \cdot d \varphi\right),
\end{gathered}
$$

and the equation for the calculation of the relative normal contact stress $\bar{\sigma}_{n F}$ for the forward slip zone can be written as follows:

$$
\begin{aligned}
\bar{\sigma}_{n F} & =e^{f \cdot\left[\left(2 m / \sqrt{m^{2}-1}\right) \cdot \operatorname{arctg}(\sqrt{(m+1) /(m-1)} \cdot \operatorname{tg}(\varphi / 2))-\varphi\right]} \cdot(1 \\
& +\int_{0}^{\varphi} e^{-f \cdot\left[\left(2 m / \sqrt{m^{2}-1}\right) \cdot \operatorname{arctg}(\sqrt{(m+1) /(m-1)} \cdot \operatorname{tg}(\varphi / 2))-\varphi\right]} \\
& \left.\cdot \frac{\sin \varphi}{m-\cos \varphi} \cdot d \varphi\right) .
\end{aligned}
$$

The analytical solution of (74) and (75) is problematic because the integrals are very complicated and has no primitive function. Therefore, it is necessary to solve integrals $I_{F}$ and $I_{B}$ using numerical methods:

$$
\begin{array}{r}
I_{B}=\int_{\varphi}^{\alpha} e^{f \cdot F_{1}(\varphi)} \cdot F_{2}(\varphi) \cdot d \varphi, \\
\text { respectively } I_{F}=\int_{0}^{\varphi} e^{-f \cdot F_{1}(\varphi)} \cdot F_{2}(\varphi) \cdot d \varphi .
\end{array}
$$

In (6) the first integral is calculated analytically from (36) and second integral is calculated numerically from (45). This combined method for solving differential equations can be called as analytical-numerical method for solving differential equation of the rolling process described in (1).

3.3. Calculation of Neutral Section. The two auxiliary constants $a_{0}$ and $a_{1}$ were used for reducing of calculated time of function $F_{1}(\varphi)$ :

$$
\begin{aligned}
& a_{0}=\frac{2 m}{\sqrt{m^{2}-1}}, \\
& a_{1}=\sqrt{\frac{m+1}{m-1}} .
\end{aligned}
$$




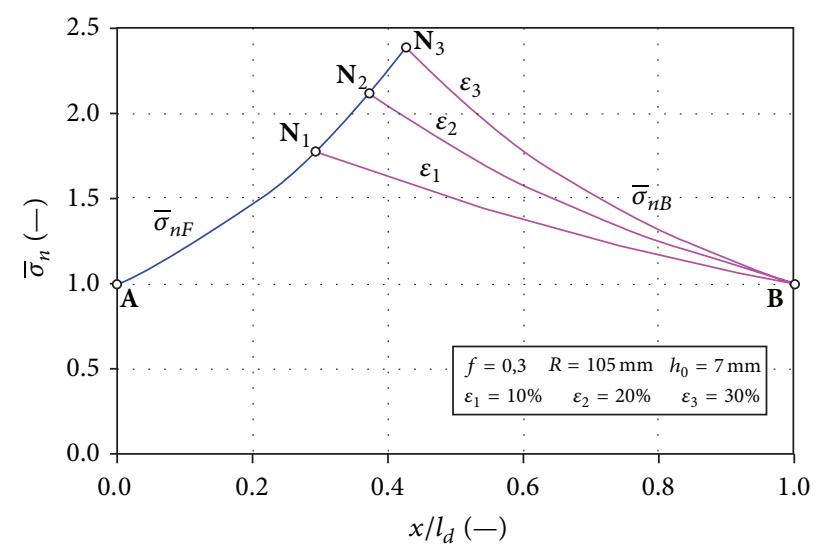

FIGURE 9: The distribution of the relative normal contact stress in rolling gap.

Equation (65) is considerably simplified after the introduction of constants:

$$
F_{1}(\varphi)=a_{0} \cdot \operatorname{arctg}\left(a_{1} \cdot \operatorname{tg} \frac{\varphi}{2}\right)-\varphi .
$$

The numerical integration was used for calculation of definite integrals described by (74) and (75).

Graphical visualization of the distribution of the relative normal contact stress in rolling gap depends on relative coordinate $x / l_{d}$ and relative deformation is given in Figure 9 . From graphical dependence the result is that neutral point $\mathbf{N}$ is significantly dependent on the relative deformation. Material output from the rolling gap is represented by a point A and the angle coordinate $\varphi=0$. Material input to the rolling gap is represented by a point $\mathbf{B}$ and the angle coordinate $\varphi=\alpha$. The influence of friction coefficient on position of neutral point and distribution of the relative normal contact stress in rolling gap is given in Figure 10 with constant value of relative deformation $(\varepsilon=20 \%)$. If the friction coefficient is increased, the peak value of relative normal contact stress is raising but weak changes were observed in shifting of neutral point depending on relative coordinate $x / l_{d}$. The influence of roll radius on position of neutral point and distribution of the relative normal contact stress in rolling gap is given in Figure 11 with constant value of relative deformation and friction coefficient. Significant influence of the roll radius to the peak value of the relative normal contact stress was observed but weak effect was observed in shifting of neutral point depending on relative coordinate $x / l_{d}$.

Determination of the neutral point $\mathbf{N}$ described by coordinate $\alpha_{n}$ is based on equality of the relative normal contact stress in backward and forward slip zone as follows:

$$
\bar{\sigma}_{n F}\left(\alpha_{n}\right)=\bar{\sigma}_{n B}\left(\alpha_{n}\right) .
$$

Substituting (74) and (75) into (79) will obtain the formula

$$
\begin{aligned}
& e^{f \cdot F_{1}\left(\alpha_{n}\right)} \cdot\left(1+\int_{0}^{\alpha_{n}} e^{-f \cdot F_{1}(\varphi)} \cdot F_{2}(\varphi) \cdot d \varphi\right) \\
& =e^{-f \cdot F_{1}\left(\alpha_{n}\right)} \cdot\left(e^{f \cdot F_{1}(\alpha)}+\int_{\alpha_{n}}^{\alpha} e^{f \cdot F_{1}(\varphi)} \cdot F_{2}(\varphi) \cdot d \varphi\right),
\end{aligned}
$$

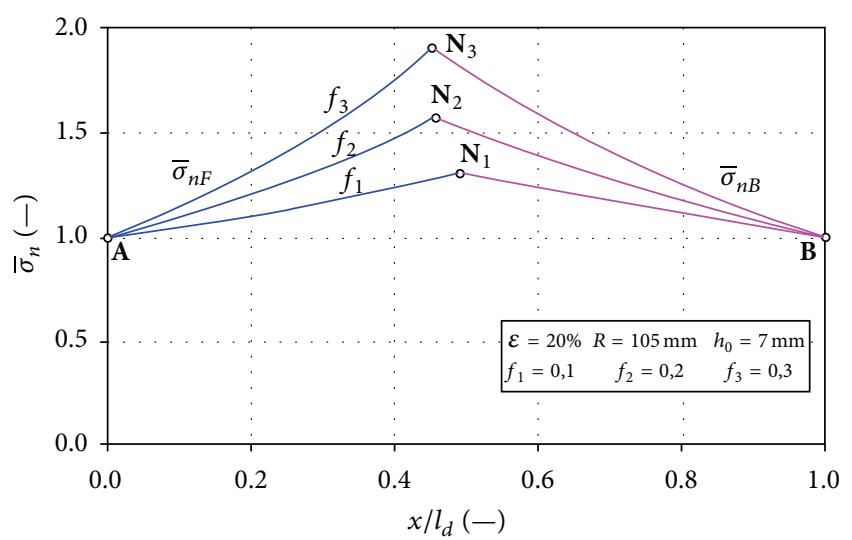

FIGURE 10: The distribution of the relative normal contact stress in rolling gap.

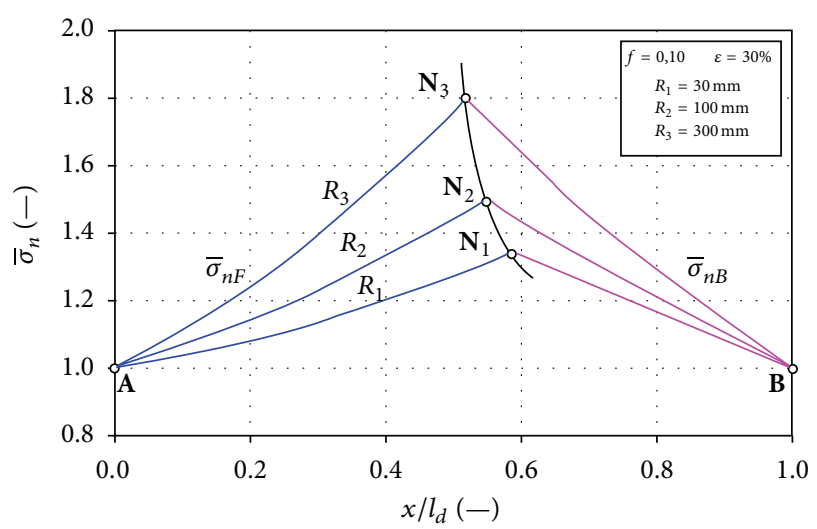

FIGURE 11: The distribution of the relative normal contact stress in rolling gap.

where function $F_{1}(\varphi)$ is given by (65) and function $F_{2}(\varphi)$ is given by (66). The coordinate of neutral angle $\alpha_{n}$ is integration limit. The analytical determination of the angle of neutral point $\alpha_{n}$ resulting from (80) is practically impossible and therefore must be used numerical solution. The numerical solution is based on the step method where interval $\varphi \in$ $\langle 0 ; \alpha\rangle$ is scanned with the step $\Delta x$. The general solution is represented with the change of variables as follows: $\varphi \rightarrow x$ and $\bar{\sigma} \rightarrow y$. The conception of numerical solution is shown in Figure 12.

The curves of the relative normal contact stress in the surrounding of point $\mathbf{N}$ are substituted by equation of straight lines in the form $y=k x+q$. If $x=0$ then coordinates of points will be $\left[y_{B 1} ; 0\right]$ and $\left[y_{F 1} ; 0\right]$. The slope of the straight lines $(f$ and $b)$ in interval $\langle x ; x+\Delta x\rangle$ can be described as follows:

$$
\begin{aligned}
& k_{F}=\frac{y_{F 2}-y_{F 1}}{\Delta x}, \\
& k_{B}=\frac{y_{B 2}-y_{B 1}}{\Delta x} .
\end{aligned}
$$


TABLE 1: The convergence of calculation of neutral angle $\alpha_{n}$.

\begin{tabular}{ccccccc}
\hline$i$ & $\begin{array}{c}\Delta \varphi_{i} \\
(\mathrm{rad})\end{array}$ & $\begin{array}{c}\alpha_{n, i} \\
(\mathrm{rad})\end{array}$ & $\begin{array}{c}\alpha_{n, i}-\alpha_{n, i-1} \\
(\mathrm{rad})\end{array}$ & $\begin{array}{c}\bar{\sigma}_{n B, i} \\
(-)\end{array}$ & $\begin{array}{c}\bar{\sigma}_{n F, i} \\
(-)\end{array}$ & $\begin{array}{c}\bar{\sigma}_{n F, i}-\bar{\sigma}_{n B, i} \\
(-)\end{array}$ \\
\hline 1 & 0,05 & 0,069778523 & - & 2,491510075 & 2,515040042 & 0,023529966 \\
2 & 0,04 & 0,069753994 & 0,000024530 & 2,492286403 & 2,514257232 & 0,021970830 \\
3 & 0,03 & 0,069524688 & 0,000229306 & 2,499555775 & 2,506948744 & 0,007392969 \\
4 & 0,02 & 0,069483702 & 0,000040985 & 2,500857391 & 2,505644227 & 0,004786837 \\
5 & 0,01 & 0,069413549 & 0,000070153 & 2,503086943 & 2,503412591 & 0,000325648 \\
6 & 0,005 & 0,069410604 & 0,000002946 & 2,503180603 & 2,503318922 & 0,000138320 \\
7 & 0,001 & 0,069408614 & 0,000001990 & 2,503243873 & 2,503255651 & 0,000011778 \\
8 & 0,0005 & 0,069408458 & 0,000000156 & 2,503248840 & 2,503250683 & 0,000001843 \\
9 & 0,0001 & 0,069408429 & 0,000000001 & 2,503249743 & 2,503249781 & 0,000000038 \\
\hline
\end{tabular}

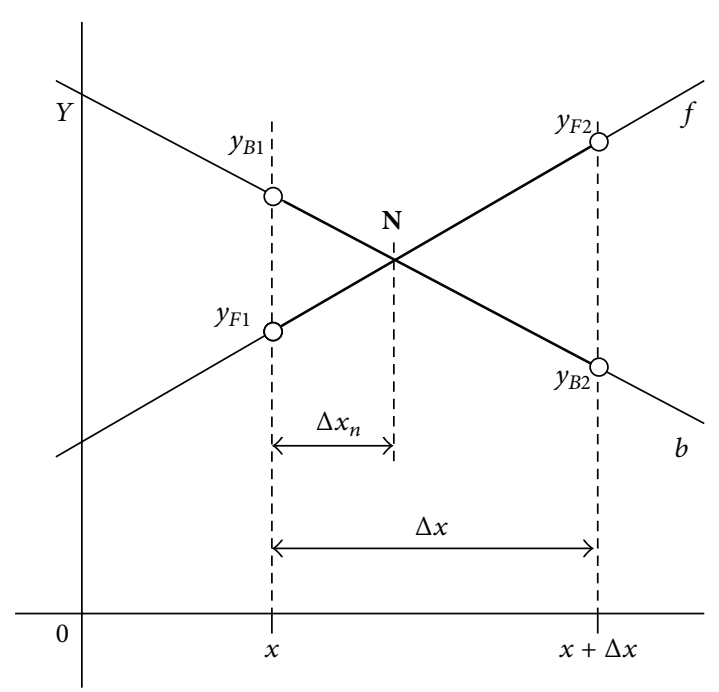

FIGURE 12: The neutral point.

Equations of the straight lines in interval $\langle x ; x+\Delta x\rangle$ can be described as follows:

$$
\begin{aligned}
& y_{F}=\frac{y_{F 2}-y_{F 1}}{\Delta x} \cdot x+y_{F 1}, \\
& y_{B}=\frac{y_{B 2}-y_{B 1}}{\Delta x} \cdot x+y_{B 1} .
\end{aligned}
$$

By comparing the equations of the straight lines $y_{F}=y_{B}$ are obtained refined local coordinate of the neutral point $\Delta x_{n}$ :

$$
\Delta x_{n}=\frac{y_{B 1}-y_{F 1}}{y_{F 2}-y_{B 2}+y_{B 1}-y_{F 1}} \cdot \Delta x .
$$

The improved value of the angle of neutral point $\mathbf{N}\left(\alpha_{n}\right)$ will be obtained from formula

$$
x_{n}=x+\Delta x_{n}
$$

The calculation of the neutral angle according to the described algorithm was performed for the following rolling conditions: roll radius $R=105 \mathrm{~mm}$, thickness of material before rolling $h_{0}=7 \mathrm{~mm}$, relative deformation $\varepsilon=$ $30 \%$, and friction coefficient $f=0,3$. Result convergence of

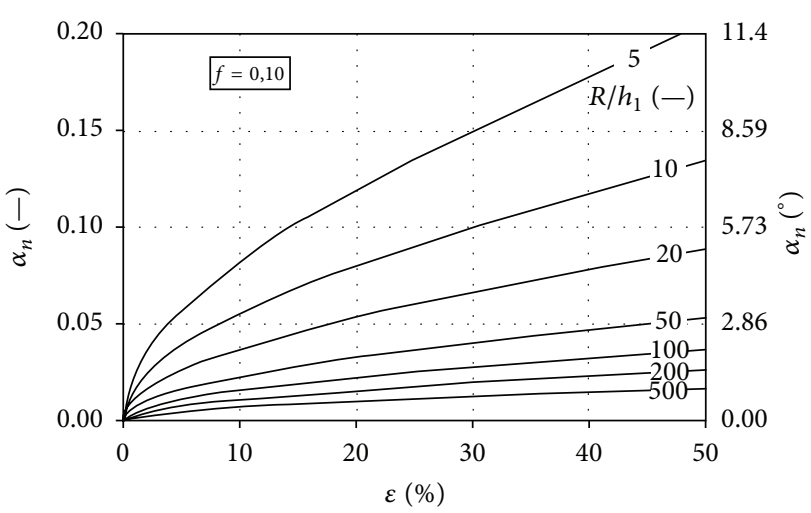

FIGURE 13: The dependence of angle of neutral point on relative deformation and ratio $R / h_{1}$.

calculation of neutral angle is given in Table 1 . The precision of calculation is evaluated by difference of values $\left(\bar{\sigma}_{n F, i}-\right.$ $\left.\bar{\sigma}_{n B, i}\right)$. Graphical visualization of the angle of neutral point in dependence on relative deformation and ratio $R / h_{1}$ is given in Figure 13. Work hardening of rolled material is not considered. The neutral angle increases with rising of relative deformation and decreasing of the ratio $R / h_{1}$. Approximate formula for ratio $\alpha_{n} / \alpha \doteq 0,5$ was presented by the authors of [16].

The validity of formula $\alpha_{n} / \alpha \doteq 0,5$ is shown in Figure 14 where ratio $\alpha_{n} / \alpha$ depends on relative deformation and ratio $R / h_{1}$. The local maximum is significantly dependent on the ratio $R / h_{1}$.

The material thickness in neutral point can be determined by the neutral angle as follows:

$$
h_{n}=h_{1}+2 R\left(1-\cos \alpha_{n}\right) \text {. }
$$

Dividing (85) by output thickness $h_{1}$ the ratio $h_{n} / h_{1}$ can be obtained in the form

$$
\frac{h_{n}}{h_{1}}=1+\frac{2 R}{h_{1}}\left(1-\cos \alpha_{n}\right) .
$$

Graphical visualization ratio $h_{n} / h_{1}$ in dependence on relative deformation and ratio $R / h_{1}$ for friction coefficient $f=0,1$ is shown in Figure 15. The ratio $h_{n} / h_{1}$ increases with rising of relative deformation and decreasing of the ratio $R / h_{1}$. 


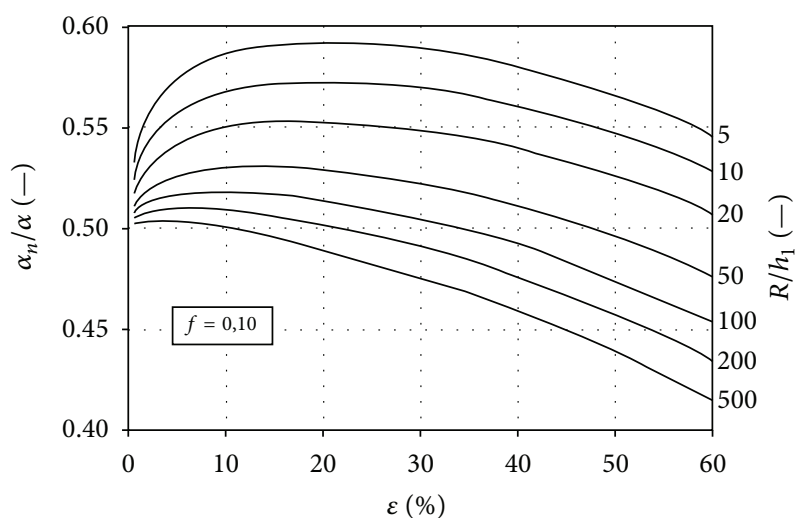

FIGURE 14: Dependence of ratio angle of neutral point and gripping angle on relative deformation and ratio $R / h_{1}$.

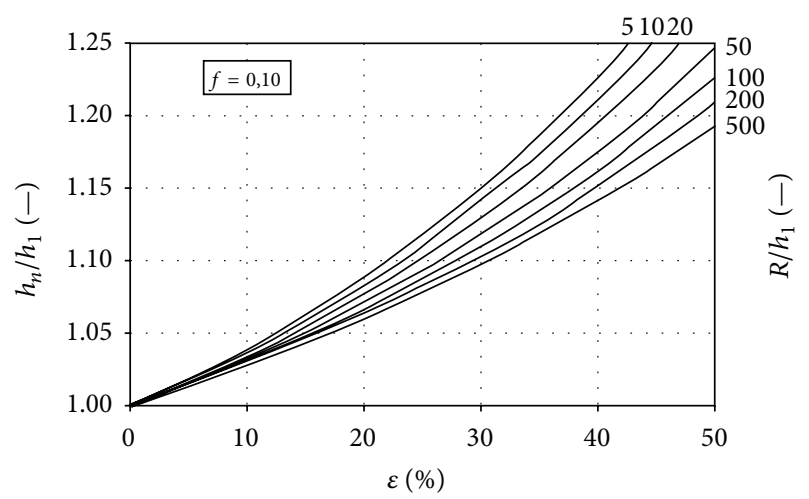

FIGURE 15: Graphical visualization of ratio $h_{n} / h_{1}$.

If the coefficient of friction $f=0$ so the status can be named as the ideal rolling. This condition can be used for simplified solution of differential equation (4) when it is possible to continue in solving (80) as follows:

$$
\int_{0}^{\alpha_{n}} F_{2}(\varphi) \cdot d \varphi=\int_{\alpha_{n}}^{\alpha} F_{2}(\varphi) \cdot d \varphi
$$

The value of variable of neutral angle $\alpha_{n}$ is one of integration limit of function $F_{2}(\varphi)$-equation (54). The calculation of integral using substitution and their differential will be as follows:

$$
\begin{aligned}
t & =m-\cos \varphi, \\
d t & =\sin \varphi \cdot d \varphi, \\
I & =\int \frac{\sin \varphi}{m-\cos \varphi} \cdot d \varphi=\int \frac{d t}{t}=\ln (t) \\
& =\ln (m-\cos \varphi) .
\end{aligned}
$$

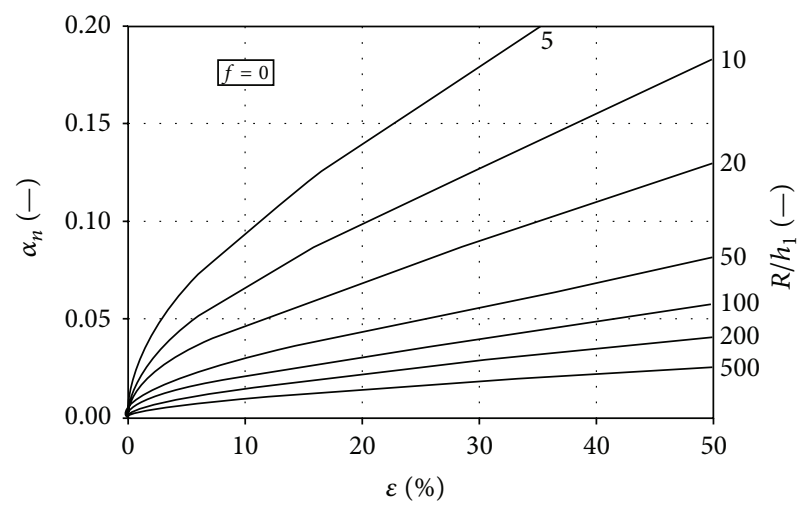

FIGURE 16: Neutral angle dependence on deformation and ratio $R / h_{1}$.

Integration in limits $0 \leq \varphi \leq \alpha_{n}$ for forward slip zone obtains the form

$$
\begin{aligned}
I_{F} & =\int_{0}^{\alpha_{n}} \frac{\sin \varphi}{m-\cos \varphi} \cdot d \varphi=[\ln (m-\cos \varphi)]_{0}^{\alpha_{n}} \\
& =\ln \left(m-\cos \alpha_{n}\right)-\ln (m-\cos 0) \\
& =\ln \frac{m-\cos \alpha_{n}}{m-1}
\end{aligned}
$$

and for backward slip zone in limits $\alpha_{n} \leq \varphi \leq \alpha$

$$
\begin{aligned}
I_{B} & =\int_{\alpha_{n}}^{\alpha} \frac{\sin \varphi}{m-\cos \varphi} \cdot d \varphi=[\ln (m-\cos \varphi)]_{\alpha_{n}}^{\alpha} \\
& =\ln (m-\cos \alpha)-\ln \left(m-\cos \alpha_{n}\right) \\
& =\ln \frac{m-\cos \alpha}{m-\cos \alpha_{n}} .
\end{aligned}
$$

The neutral angle of ideal rolling process can be calculated from comparison of (89) and (90) as follows:

$$
\cos \alpha_{n}=m-\sqrt{(m-1) \cdot(m-\cos \alpha)} .
$$

Substituting (51) to (91) and using simplification $\cos \alpha \approx R / h_{1}$ the next formula will be obtained:

$$
\alpha_{n}=\arccos \left(1+\left(1-\frac{1}{\sqrt{1-\varepsilon}}\right) \frac{h_{1}}{2 R}\right) .
$$

Graphical visualization equation (92) is given in Figure 16. The neutral angle is increased with rising of relative deformation and decreasing of ratio $R / h_{1}$. From comparison of dependences shown in Figure $13(f=0,1)$ Figure $16(f=0)$ is the result that neutral angle is slightly higher if friction coefficient $f=0$. Equation (92) can be considered as simplest form for direct calculation of neutral angle with satisfying results.

3.4. Calculation of Average Normal Contact Stress. The distribution of relative normal contact stress to rolls in plastic deformation zone is shown in Figures 9, 10, and 11 by the 


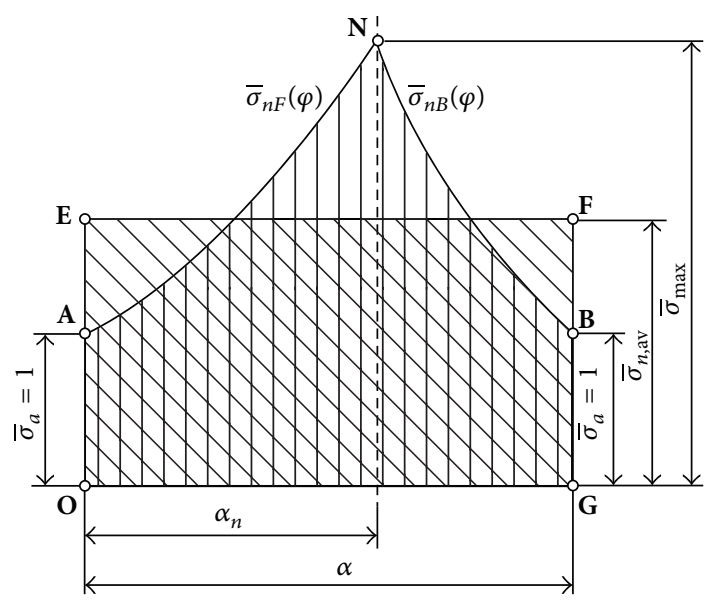

FIGURE 17: The average relative normal contact stress $\bar{\sigma}$.

curves ANB. The rolling force can be calculated from the average relative normal contact stress $\bar{\sigma}_{n \text {,av }}$ along gripping angle $\alpha$ (surface of rectangular $\mathbf{O E F G O}$ ) as is given in Figure 17.

It is supposed the egality of surfaces OANBGO $\equiv$ OEFGO. If it is valid that $\bar{\sigma}=\bar{\sigma}_{n, \text { av }}$ then the following can be written:

$$
\bar{\sigma}=\frac{1}{\alpha-0} \cdot \int_{0}^{\alpha} \bar{\sigma}_{n}(\varphi) \cdot d \varphi .
$$

The calculation of $\bar{\sigma}$ must be done independently for backward and forward slip zone according to the following formula:

$$
\bar{\sigma}=\frac{1}{\alpha} \cdot\left[\int_{0}^{\alpha_{n}} \bar{\sigma}_{n F}(\varphi) \cdot d \varphi+\int_{\alpha_{n}}^{\alpha} \bar{\sigma}_{n B}(\varphi) \cdot d \varphi\right] .
$$

Substituting (74) and (75) to (94) is obtained in the following form:

$$
\begin{aligned}
\bar{\sigma}= & \frac{1}{\alpha} \cdot\left[\int _ { 0 } ^ { \alpha _ { n } } \left(e^{f \cdot F_{1}(\varphi)}\right.\right. \\
& \left.\cdot\left(1+\int_{0}^{\varphi} e^{-f \cdot F_{1}(\varphi)} \cdot F_{2}(\varphi) \cdot d \varphi\right)\right) \cdot d \varphi \\
& +\int_{\alpha_{n}}^{\alpha}\left(e^{-f \cdot F_{1}(\varphi)}\right. \\
& \left.\left.\cdot\left(e^{f \cdot F_{1}(\alpha)}+\int_{\varphi}^{\alpha} e^{f \cdot F_{1}(\varphi)} \cdot F_{2}(\varphi) \cdot d \varphi\right)\right) \cdot d \varphi\right] .
\end{aligned}
$$

The limits of integration for a forward slip zone are defined by interval $\varphi \in\left\langle 0 ; \alpha_{n}\right\rangle$ and calculation of $\bar{\sigma}_{n F}$ is performed according to (75). Identically it is valid for backward slip zone where $\varphi \in\left\langle\alpha_{n} ; \alpha\right\rangle$ and calculation of $\bar{\sigma}_{n B}$ is performed according to (74). The definite integrals in (95) can be performed by some numerical mathematical method. Graphical visualization of (1) is given in Figure 18. Function $\bar{\sigma}$ throughout the observed interval is monotonically increasing. The relative normal contact stress is increased with rising of relative

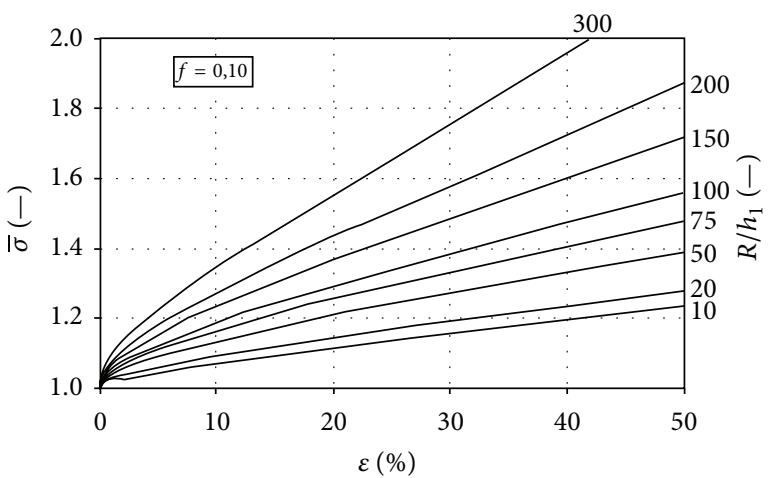

FIGURE 18: The dependence of function $\bar{\sigma}$ on relative deformation $\varepsilon$ and ratio $R / h_{1}$.

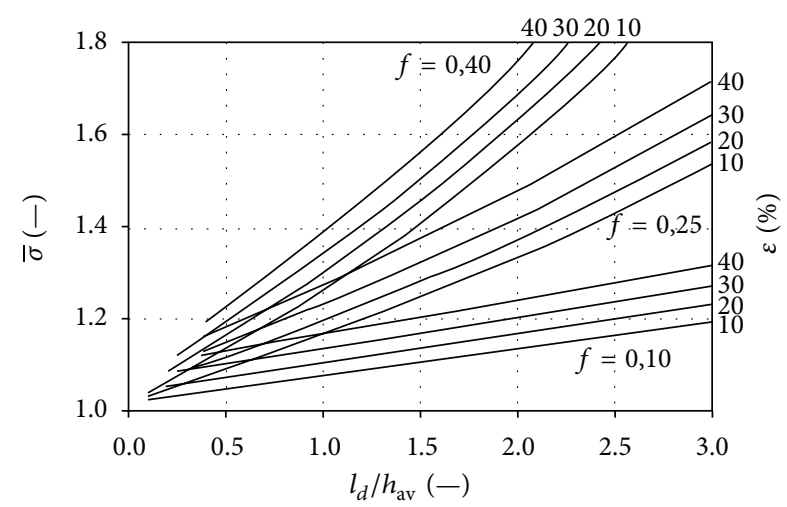

FIGURE 19: The dependence of function $\bar{\sigma}$ on ratio $l_{d} / h_{\mathrm{av}}$ and relative deformation.

deformation and decreasing of ratio $R / h_{1}$. Dependence of relative normal contact stress on ratio $l_{d} / h_{\mathrm{av}}$ and relative deformation for three groups of friction coefficient are given in Figure 19. From dependence the result is that the strongest effect on relative normal contact stress has friction coefficient.

For numerical integration of (95) Simpson's quadrature rule can be used. The author of [24] presented Weddle formula which is more effective than Simpson's quadrature rule for numerical calculation of general function $f(x)$. The advantage of this numerical method is faster convergence of calculation. The continuous function $f(x)$ is numerically integrated in interval $x \in\langle a ; b\rangle$ where $b>a$ which is divided on final number of subinterval with step $h$ as follows:

$$
h=\frac{b-a}{n},
$$

where $n$ is the whole positive number:

$$
\begin{aligned}
& \int_{a}^{b} f(x) \cdot d x=\frac{3}{10} h\left(y_{0}+5 y_{1}+y_{2}+6 y_{3}+y_{4}+5 y_{5}\right. \\
& \quad+2 y_{6}+5 y_{7}+y_{8}+6 y_{9}+y_{10}+5 y_{11}+2 y_{12}+5 y_{13} \\
& \left.+y_{14}+\cdots+6 y_{n-3}+y_{n-2}+5 y_{n-1}+y_{n}\right),
\end{aligned}
$$

where $y_{0}=f(a), y_{1}=f(a+h), y_{2}=f(a+2 h), y_{3}=$ $f(a+3 h), \ldots, y_{i}=f(a+i \cdot h)$, and $y_{n}=f(b), i$ is the 
TABLE 2: The convergence of calculation of $\bar{\sigma}$ function according to Weddle formula.

\begin{tabular}{cccc}
\hline$i$ & $n$ & $\bar{\sigma}_{n B, i}$ & $\bar{\sigma}_{n B, i}-\bar{\sigma}_{n B, i-1}$ \\
\hline 1 & 6 & 4,116857469 & - \\
2 & 12 & 4,116875359 & $-0,000017889$ \\
3 & 18 & 4,116875502 & $-0,000000143$ \\
4 & 24 & 4,116875512 & $-0,000000010$ \\
5 & 30 & 4,116875514 & $-0,000000002$ \\
6 & 36 & 4,116875514 & 0,000000000 \\
\hline
\end{tabular}

whole positive number $(i=1,2,3, \ldots, n)$. The calculation of relative normal contact stress in backward slip zone-equation (66) according to Weddle formula is given in Table 2. The precision of calculation is evaluated according to difference of values: $\bar{\sigma}_{n B, i}-\bar{\sigma}_{n B, i-1}$. The satisfied precision of calculation $\bar{\sigma}_{n B, i}$ is obtained after $n=18$ where $\left(\bar{\sigma}_{n B, i}-\bar{\sigma}_{n B, i-1}\right) \approx 1.10^{-6}$.

Boundary conditions of rolling process were given as follows: relative deformation $\varepsilon=60 \%$, ratio $R / h_{1}=10$, friction coefficient $f=0,2$, and angle coordinate $\varphi=0,05 \mathrm{rad}$. From comparison of numerical calculations the result is that numerical integration according to Weddle formula is four times faster than numerical integration realized according to Simpson's quadrature rule.

The previous calculations were carried out provided without work hardening of material during rolling process.

In the next part the rolling process with acceptance of isotropic work hardening of material will be described. Alexander [25] mentions Swift described work hardening curves according to formula

$$
Y S=Y S_{0} \cdot(1+B \cdot \bar{\varepsilon})^{n}
$$

where $Y S$ is the uniaxial yield stress from static tensile test depending on true strain $\bar{\varepsilon}=\ln \left(h_{0} / h_{1}\right), Y S_{0}$ is the uniaxial yield stress from static tensile test resulting from annealing state of material, and $B, n$ is the material constants.

The average value of flow stress can be calculated as follows:

$$
\sigma_{a, \mathrm{av}}=\frac{1}{\bar{\varepsilon}_{2}-\bar{\varepsilon}_{1}} \int_{\bar{\varepsilon}_{1}}^{\bar{\varepsilon}_{2}} \sigma_{a} d \bar{\varepsilon},
$$

where $\bar{\varepsilon}_{1}$ is the true strain before actual rolling and $\bar{\varepsilon}_{2}$ is the final true strain after rolling.

For calculation of flow stress the author of [25] used the formula in which effective stress and effective strain are given:

$$
\sigma_{a}=\frac{2}{\sqrt{3}} \cdot Y S_{0} \cdot\left(1+\frac{2}{\sqrt{3}} \cdot B \cdot \bar{\varepsilon}\right)^{n} .
$$

Substituting (100) into (99) the following is obtained:

$$
\sigma_{a, \mathrm{av}}=\frac{1}{\bar{\varepsilon}_{2}-\bar{\varepsilon}_{1}} \int_{\bar{\varepsilon}_{1}}^{\bar{\varepsilon}_{2}} \frac{2}{\sqrt{3}} \cdot Y_{0} \cdot\left(1+\frac{2}{\sqrt{3}} \cdot B \cdot \bar{\varepsilon}\right)^{n} \cdot d \bar{\varepsilon},
$$

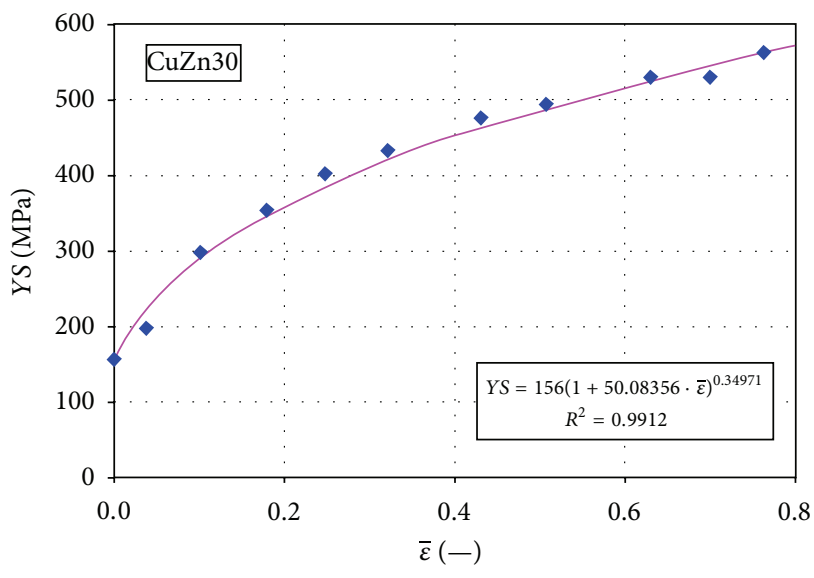

FiguRE 20: Uniaxial stress-strain curve for brass CuZn30.

and after integration average value of flow stress has a form

$$
\begin{aligned}
& \sigma_{a, \mathrm{av}}=\frac{Y S_{0}}{(n+1) \cdot\left(\bar{\varepsilon}_{2}-\bar{\varepsilon}_{1}\right) \cdot B}\left[\left(1+\frac{2}{\sqrt{3}} \cdot B \cdot \bar{\varepsilon}_{2}\right)^{n+1}\right. \\
&\left.-\left(1+\frac{2}{\sqrt{3}} \cdot B \cdot \bar{\varepsilon}_{1}\right)^{n+1}\right] .
\end{aligned}
$$

Let us assume that material is processing by rolling from annealed state for which $\bar{\varepsilon}_{1}=0$ is valid and transform upper limit of integral $\bar{\varepsilon}_{2}=\bar{\varepsilon}$, (102) is simplified to form

$$
\sigma_{a, \mathrm{av}}=\frac{Y S_{0}}{(n+1) \cdot \bar{\varepsilon} \cdot B}\left[\left(1+\frac{2}{\sqrt{3}} \cdot B \cdot \bar{\varepsilon}\right)^{n+1}-1\right] .
$$

The measurements values of yield stress depending on true strain of laboratory cold rolling experiment are given in Figure 20. For approximation of measurement values of stress-strain curve (98) was used. Final regression equation of stress-strain curve for brass CuZn30 has a form

$$
Y S=156 \cdot(1+50,08356 \cdot \bar{\varepsilon})^{0,34971} .
$$

Graphical visualization of (104) is given in Figure 20.

Substituting (104) into (103) the following formula is obtained:

$$
\sigma_{a, \mathrm{av}}=\frac{2,30775}{\bar{\varepsilon}}\left[(1+57,83152 \cdot \bar{\varepsilon})^{1,34971}-1\right] .
$$

Measured and calculated data from experimental cold rolling schedule are given in Table 3. The calculation of deformation resistance was carried out according to formula

$$
\sigma_{D}=\frac{F_{r}}{S}
$$

where $F_{r}$ is the rolling force and $S$ is the contact surface of rolled material with roll.

The average relative normal contact stress calculated from measurement data is given as follows:

$$
\bar{\sigma}_{\text {meas }}=\frac{\sigma_{D}}{\sigma_{a, \mathrm{av}}} .
$$


TABLE 3: Measured and calculated data from experimental cold rolling schedule.

\begin{tabular}{lcccccccccc}
\hline Number & $\begin{array}{c}\varepsilon \\
(\%)\end{array}$ & $\begin{array}{c}\bar{\varepsilon} \\
(-)\end{array}$ & $\begin{array}{c}R / h_{1} \\
(-)\end{array}$ & $\begin{array}{c}S \\
\left(\mathrm{~mm}^{2}\right)\end{array}$ & $\begin{array}{c}F_{r} \\
(\mathrm{kN})\end{array}$ & $\begin{array}{c}\sigma_{D} \\
(\mathrm{MPa})\end{array}$ & $\begin{array}{c}\sigma_{a, \mathrm{av}} \\
(\mathrm{MPa})\end{array}$ & $\begin{array}{c}\bar{\sigma}_{\text {meas }} \\
(-)\end{array}$ & $\begin{array}{c}\bar{\sigma} \\
(-)\end{array}$ & $\begin{array}{c}\bar{\sigma}_{\text {meas }}-\bar{\sigma} \\
(-)\end{array}$ \\
\hline 1 & 10,39 & 0,1097 & 34,77 & 157,10 & 65 & 413,76 & 279,41 & 1,431 & 1,251 & 0,180 \\
2 & 17,46 & 0,1918 & 37,63 & 230,18 & 110 & 477,89 & 325,40 & 1,423 & 1,371 & 0,052 \\
3 & 22,69 & 0,2573 & 40,54 & 247,76 & 137 & 552,96 & 355,38 & 1,512 & 1,468 & 0,044 \\
4 & 27,38 & 0,3199 & 43,03 & 280,69 & 165 & 587,84 & 380,55 & 1,506 & 1,560 & $-0,054$ \\
5 & 30,06 & 0,3575 & 44,68 & 317,00 & 178 & 561,51 & 394,41 & 1,390 & 1,617 & $-0,226$ \\
\hline
\end{tabular}

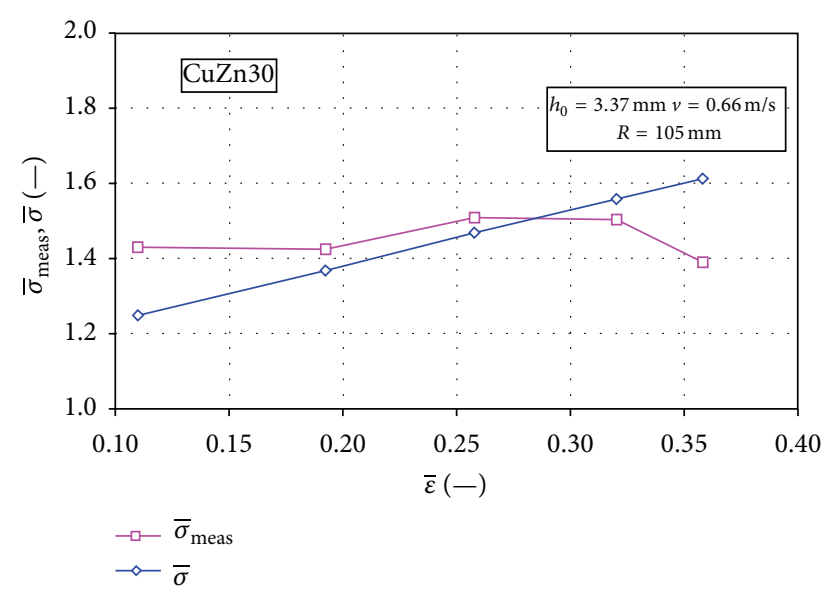

FIGURE 21: Uniaxial stress-strain curve for brass CuZn30.

Average value of flow stress is calculated according to (105). Theoretical value of $\bar{\sigma}$ function is calculated according to (95) with friction coefficient $f=0,2$. Numeric comparison of measured and theoretically calculated values of $\bar{\sigma}$ function is given in Table 3 and graphical comparison is shown in Figure 21.

The differences between measured and calculated values of the average relative normal contact stress are from interval $\Delta \in\langle 3 ; 14\rangle[\%]$. These deviations are resulting from the calculation which does not take into account the flattening rolls.

3.5. Calculation of Front and Back Tensions. Back tension is actuating in direction of $x$-axis and marked by label $\sigma_{x 0}$. Front tension is actuating in direction of $x$-axis and marked by label $\sigma_{x 1}$. According to Figure 1 point $\mathbf{B}$ is valid: $x=l_{d}$ and tension stress $\sigma_{x}=\sigma_{x 0}$. The tension stresses $\sigma_{x 0}$ and $\sigma_{x 1}$ can be described by using flow stress as follows:

$$
\begin{aligned}
& \sigma_{x 0}=\xi_{0} \cdot \sigma_{p}, \\
& \sigma_{x 1}=\xi_{1} \cdot \sigma_{p},
\end{aligned}
$$

where $\xi_{0}$ and $\xi_{1}$ are coefficient for back and front tensions, $0 \leq \xi_{0}<1$ (for without the back tension $\xi_{0}=0$ and for maximal back tension have to be valid $\xi_{0}<1$ ), and $0 \leq \xi_{1}<1$ (for without the front tension $\xi_{1}=0$ and for maximal front tension have to be valid $\xi_{1}<1$ ).
Using the Tresca condition of plasticity formulae of plasticity of material for backward and forward slip zones will be obtained as follows:

$$
\begin{aligned}
& \sigma_{n B}-\left(-\sigma_{x 0}\right)=\sigma_{n B}+\sigma_{x 0}=\sigma_{p}, \\
& \sigma_{n F}-\left(-\sigma_{x 1}\right)=\sigma_{n F}+\sigma_{x 1}=\sigma_{p} .
\end{aligned}
$$

Editing of (109) is received:

$$
\begin{aligned}
\frac{\sigma_{n B}}{\sigma_{a}} & =\frac{\sigma_{a}}{\sigma_{a}}-\frac{\xi_{0} \sigma_{a}}{\sigma_{a}} \\
& \Longrightarrow \bar{\sigma}_{n B}=1-\xi_{0}, \\
\frac{\sigma_{n F}}{\sigma_{a}} & =\frac{\sigma_{a}}{\sigma_{a}}-\frac{\xi_{1} \sigma_{a}}{\sigma_{a}} \\
& \Longrightarrow \bar{\sigma}_{n F}=1-\xi_{1} .
\end{aligned}
$$

The integration constants $C_{B}$ and $C_{F}$ which are describing front and back tensions can be determined from limit conditions given by (111) and (113). The integration constant $C_{F}$ for forward slip zone and for $\varphi=0$ resulting from (67) will be as follows:

$$
C_{F}=1-\xi_{1} .
$$

The integration constant $C_{B}$ for backward slip zone and for $\varphi=\alpha$ resulting from (66) will be as follows:

$$
C_{B}=\left(1-\xi_{0}\right) \cdot e^{f \cdot F_{1}(\alpha)} .
$$

The formula for calculation of relative normal contact stress at forward slip zone with consideration of front tension will be as follows:

$$
\begin{aligned}
\bar{\sigma}_{n F}= & e^{f \cdot F_{1}(\varphi)} \\
& \cdot\left(\left(1-\xi_{1}\right)+\int_{0}^{\varphi} e^{-f \cdot F_{1}(\varphi)} \cdot F_{2}(\varphi) \cdot d \varphi\right) .
\end{aligned}
$$

And for calculation of relative normal contact stress at backward slip zone with consideration of back tension will be as follows:

$$
\begin{aligned}
\bar{\sigma}_{n B} & \\
= & e^{-f \cdot F_{1}(\varphi)} \\
& \cdot\left(\left(1-\xi_{0}\right) \cdot e^{f \cdot F_{1}(\alpha)}+\int_{\varphi}^{\alpha} e^{f \cdot F_{1}(\varphi)} \cdot F_{2}(\varphi) \cdot d \varphi\right) .
\end{aligned}
$$




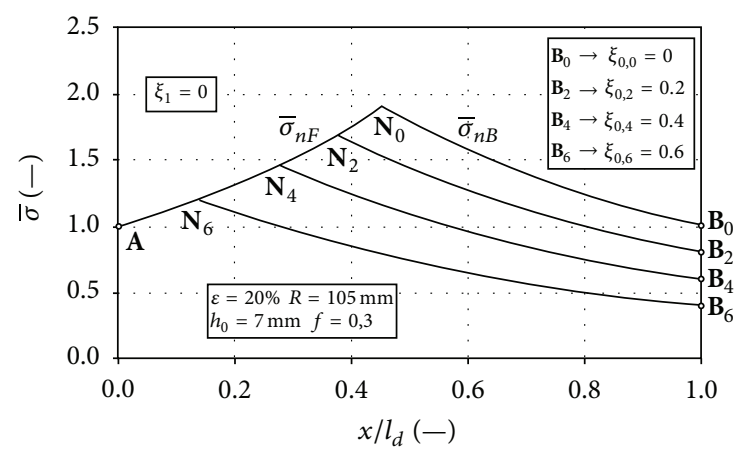

Figure 22: The dependence of function $\bar{\sigma}$ on ratio $x / l_{d}$ and back tension.

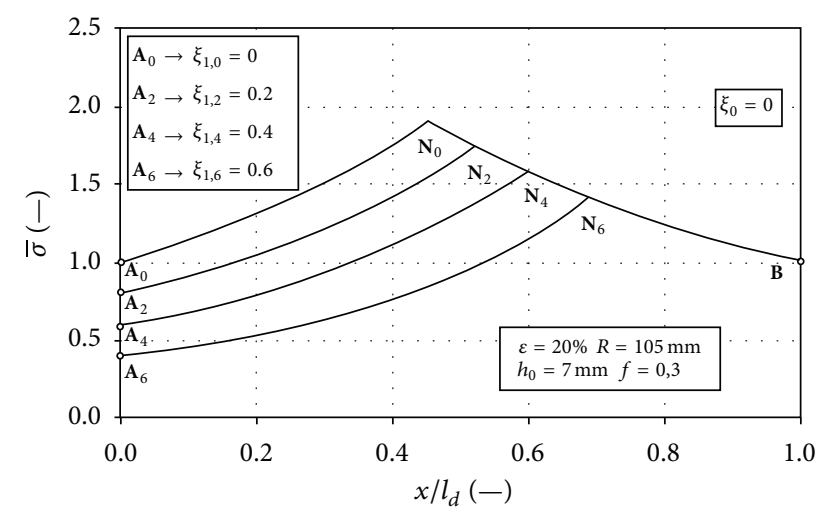

FIgURE 23: The dependence of function $\bar{\sigma}$ on ratio $x / l_{d}$ and front tension.

The function $F_{1}(\varphi)$ was described by $(65)$ and function $F_{2}(\varphi)$ by (64). The distribution of the relative normal contact stress in rolling gap depends on relative coordinate $x / l_{d}$ and back tension is given in Figure 22. From graphical dependence the result is that neutral point $\mathbf{N}$ is significantly dependent on the back tension. If coefficient of front tension is $\xi_{1}=0$ then front tension stress will be $\sigma_{x 1}=0$. The back tension stress described by flow stress will be given by coefficient $\xi_{0}$. The value of back tension is characterizing by point $\mathbf{B}$. For example, for point $\mathbf{B}_{4}$ being $\xi_{0,4}=0,4$ it means that back tension will be as follows: $\sigma_{x 0}=0,4 \sigma_{a}$.

If the back tension increases then average relative normal contact stress is decreased. The front and back tensions are not active in neutral point $\mathbf{N}_{0}$. The neutral point is shifting to exit side of rolling gap with increase of back tension.

The distribution of the relative normal contact stress in rolling gap depends on relative coordinate $x / l_{d}$ and front tension is given in Figure 23. From graphical dependence the result is that neutral point $\mathbf{N}$ is significantly dependent on the front tension. If coefficient of back tension is $\xi_{0}=0$ then back tension stress will be $\sigma_{x 0}=0$. The front tension stress described by flow stress will be given by coefficient $\xi_{1}$. The value of front tension is characterized by point $\mathbf{A}$. For example, for point $\mathbf{A}_{4}$ being $\xi_{1,4}=0,4$ it means that back tension will be as follows: $\sigma_{x 1}=0,4 \sigma_{a}$.

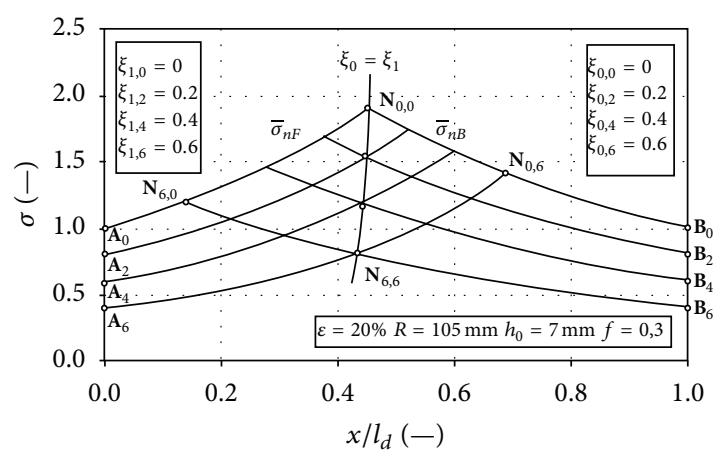

FIGURE 24: The dependence of function $\bar{\sigma}$ on ratio $x / l_{d}$ back and front tension.

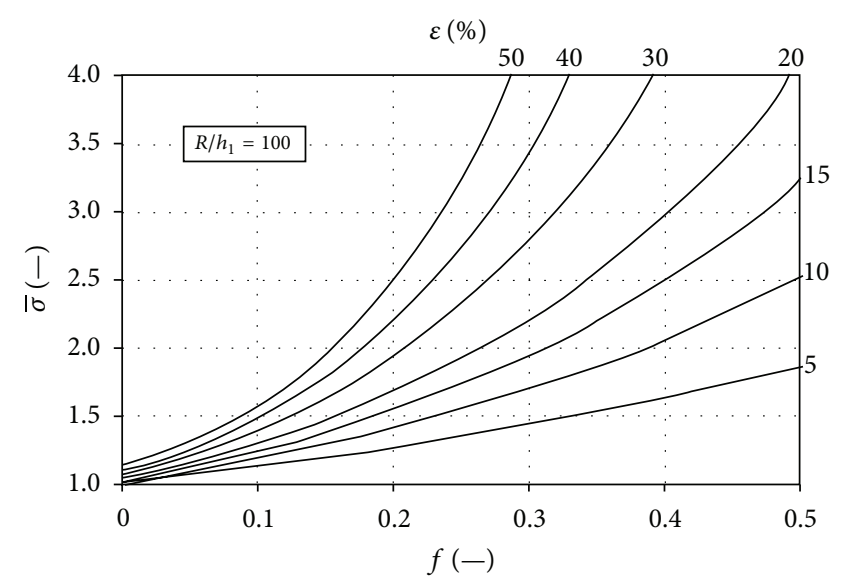

FIGURE 25: The dependence of function $\bar{\sigma}$ on friction coefficient.

The common action of back and front tension on the distribution of the relative normal contact stress in rolling gap depends on relative coordinate $x / l_{d}$ that is given in Figure 24 . The indexing of point $\mathbf{N}_{x, y}$ is as follows: $x$ is the tension stress on enter side of material to rolling gap, $y$ is the tension stress on exit side of material from rolling gap. This means that $\mathbf{N}_{0,0}$ is representing intersection point of curves without action of front and back tension: $\xi_{0}=\xi_{1}=0$; for $\mathbf{N}_{6,6}$ it is for maximal values of front and back tension: $\xi_{0}=\xi_{1}=0,6$.

3.6. Calculation of Friction Coefficient. The simple determination of friction coefficient can be used with graphical dependence given in Figure 25 for constant ratio $R / h_{1}=$ 100 . More precisely determination of friction coefficient depends on deformation resistance of rolled material. The deformation resistance of rolled material $\sigma_{D}$ is characterized by three-axial stress state and depends on friction coefficient between rolls and rolled material. In the case of action only one-axial stress state is function of friction eliminated and stress is defined as flow stress $\sigma_{a}$. If ratio $R / h_{1}$ and deformation $\varepsilon$ are constant then the relative normal contact stress in rolling gap depends only on friction coefficient. Mathematical expression describing relationship between $\sigma_{D}$ and $\sigma_{a}$ can be given as follows:

$$
\sigma_{D}=\sigma_{a} \cdot \bar{\sigma}_{0}
$$




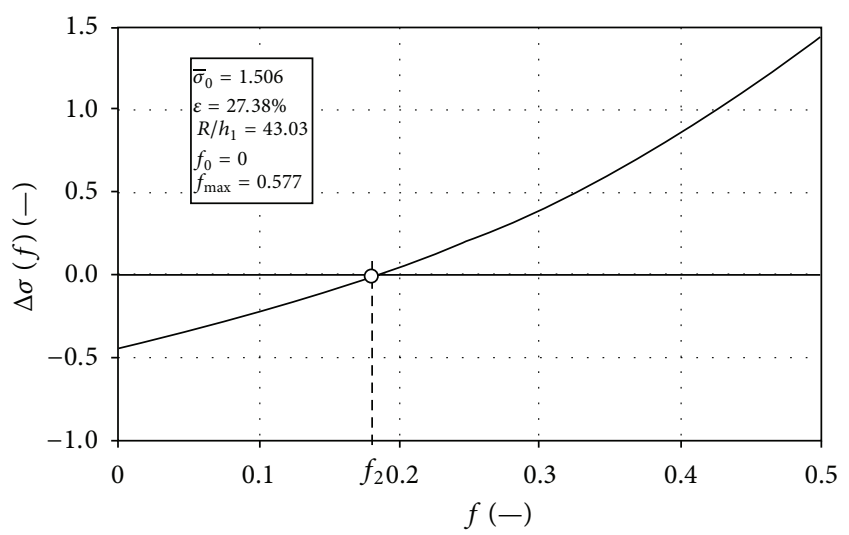

FIGURE 26: The dependence of function $\Delta \bar{\sigma}$ on friction coefficient.

From (118) the following can be determined:

$$
\bar{\sigma}_{0}=\frac{\sigma_{D}}{\sigma_{a}} .
$$

Substituting (119) into (95) and subtraction of (119) from (95) is obtained:

$$
\Delta \bar{\sigma}=\bar{\sigma}(f)-\bar{\sigma}_{0}
$$

Graphical dependence of $\Delta \bar{\sigma}$ is given in Figure 26. Intersection point of curve describing $\Delta \bar{\sigma}$ function with zero value of $x$-axis is the determining value of friction coefficient $f_{2}$. The value of friction coefficient $f_{2}$ valid for given conditions of rolling process. For numerical calculation of friction coefficient Regula Falsi method was used. Maximal value of friction coefficient results from

(i) Tresca condition of plasticity: $f_{1}=0,5$,

(ii) HMH condition of plasticity: $f_{1}=0,577$.

The first approximation for calculation of friction coefficient $f_{2}$ using Regula Falsi method will be as follows:

$$
f_{2}=\frac{f_{0} \cdot \Delta \bar{\sigma}\left(f_{1}\right)-f_{1} \cdot \Delta \bar{\sigma}\left(f_{0}\right)}{\Delta \bar{\sigma}\left(f_{1}\right)-\Delta \bar{\sigma}\left(f_{0}\right)}
$$

The calculation of friction coefficient $f_{2}$ using Regula Falsi method for rolling conditions given in Table 3; line number $4\left(\bar{\sigma}_{0}=1,506\right.$, ratio: $\left.R / h_{1}=43,03, \varepsilon=27.38 \%\right)$ is shown in Table 4. Calculation precision on three decimal positions $\left(f_{2}=0,182\right)$ was obtained after $n=7$ steps.

3.7. Calculation of Coefficient of the Arm of Rolling Force. The rolling torque of rolling process depends on the coefficient of the arm of rolling force $\psi$ and the rolling force $F_{r}$. The coefficient of the arm of rolling force is calculated according to the following formula:

$$
\psi=\frac{a}{l_{d}},
$$

where $a$ is the arm of rolling force and $l_{d}$ is the length of contact arc.
TABLE 4: The calculation of friction coefficient $f_{2}$ using Regula Falsi method.

\begin{tabular}{ccccccc}
\hline$n$ & $f_{0}$ & $f_{1}$ & $\Delta \bar{\sigma}\left(f_{0}\right)$ & $\Delta \bar{\sigma}\left(f_{1}\right)$ & $f_{2}$ & $\Delta \bar{\sigma}\left(f_{2}\right)$ \\
$(-)$ & $(-)$ & $(-)$ & $(-)$ & $(-)$ & $(-)$ \\
\hline 0 & 0 & 0,5 & $-0,441968$ & 1,433027 & 0,117858 & $-0,174444$ \\
1 & 0,117858 & 0,5 & $-0,174444$ & 1,433027 & 0,159329 & $-0,064807$ \\
2 & 0,159329 & 0,5 & $-0,064807$ & 1,433027 & 0,174069 & $-0,023184$ \\
3 & 0,174069 & 0,5 & $-0,023184$ & 1,433027 & 0,179258 & $-0,008176$ \\
4 & 0,179258 & 0,5 & $-0,008176$ & 1,433027 & 0,181077 & $-0,002869$ \\
5 & 0,181077 & 0,5 & $-0,002869$ & 1,433027 & 0,181715 & $-0,001005$ \\
6 & 0,181715 & 0,5 & $-0,001005$ & 1,433027 & 0,181938 & $-0,000352$ \\
7 & 0,181938 & 0,5 & $-0,000352$ & 1,433027 & 0,182016 & $-0,000123$ \\
8 & 0,182016 & 0,5 & $-0,000123$ & 1,433027 & 0,182043 & $-0,000043$ \\
\hline
\end{tabular}

The development of the relative normal contact stress in coordinates $\bar{\sigma}_{n}-\varphi$ is shown in Figure 17 from which the result is that surface is delimited by points OANBGO on the interval $\varphi \in\langle 0 ; \alpha\rangle$.

The resultant of the force is determined from the angle gravity centre $\varphi=\alpha_{a}$ in which the force acts. The angle gravity centre $\alpha_{a}$ of the stress surface $\mathbf{O A N B G O}$ is defined as the ratio of static moment $M_{S}$ and the stress surface $S_{\sigma}$ :

$$
\alpha_{a}=\frac{M_{S}}{S_{\sigma}} .
$$

The stress surface $S_{\sigma}$ can be determined as follows:

$$
S_{\sigma}=\alpha \cdot \bar{\sigma},
$$

where $\alpha$ is the gripping angle and $\bar{\sigma}_{n, \text { av }}$ is the average relative normal contact stress.

The static moment $M_{S}$ then can be determined as follows:

$$
M_{S}=\int_{0}^{\alpha} \varphi \cdot \sigma_{n}(\varphi) \cdot d \varphi .
$$

Result from Figure 27 can be described: forward slip zone by curve with points $\mathrm{AN}$ and angle coordinate $\varphi \in\left\langle 0 ; \alpha_{n}\right\rangle$; backward slip zone by curve with points NB and angle coordinate $\varphi \in\left\langle\alpha_{n} ; \alpha\right\rangle$. The solution of integral equation (125) is performed by numerical integration separately for backward and forward slip zone as follows:

$$
M_{S}=\int_{0}^{\alpha_{n}} \varphi \cdot \bar{\sigma}_{n F}(\varphi) \cdot d \varphi+\int_{\alpha_{n}}^{\alpha} \varphi \cdot \bar{\sigma}_{n B}(\varphi) \cdot d \varphi,
$$

where $\bar{\sigma}_{n F}(\varphi)$ is described by $(75)$ and $\bar{\sigma}_{n B}(\varphi)$ is described by (74).

The calculation of integrals is carried out by Weddle formulae [24]. Length of arm of force action is calculated using the following formula:

$$
a=R \cdot \sin \alpha_{a} .
$$

The numerical calculation and visualization of the coefficient of arm of rolling force $\psi$ depends on the relative deformation 


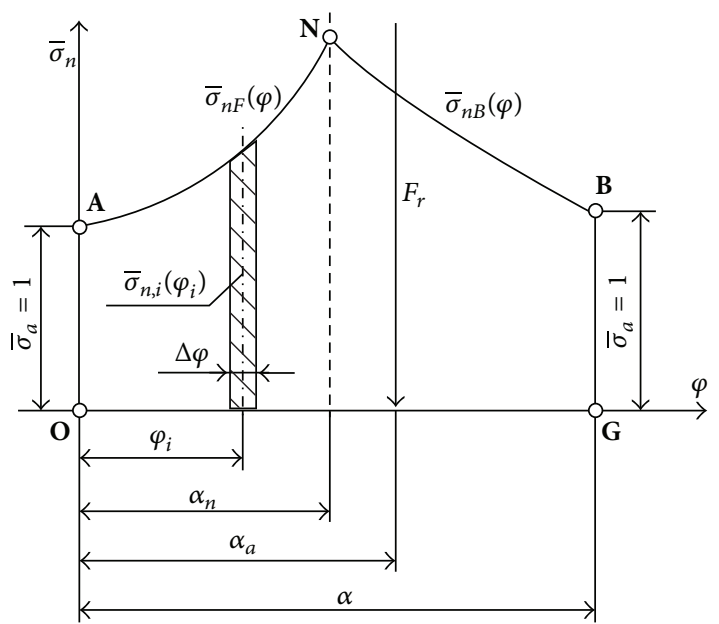

FIGURE 27: The determination of angle gravity centre $\alpha_{a}$ as a point of rolling force $F_{r}$.

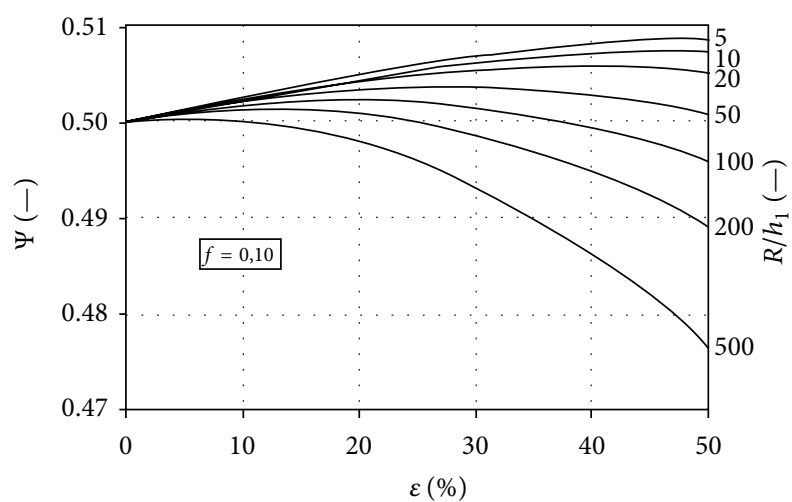

FIGURE 28: The dependence of the coefficient of arm of rolling force $\psi$ on relative deformation.

and ratio $R / h_{1}$ is shown in Figure 28. From graphical dependence the result is that the coefficient of the arm of rolling force is decreased with increase of relative deformation and ration $R / h_{1}$.

The coefficient of arm of rolling force can be determined also from the following formula [3]:

$$
\psi=\frac{\alpha_{a}}{\alpha}
$$

The authors of [3] introduce calculation of $\psi$ the following approximated formula: $\psi=\alpha_{a} / \alpha \doteq 0,5$. From the graphical dependence in Figure 28 the result is that value can be obtained only if deformation $\varepsilon=0 \%$.

3.8. Calculation of Rolling Force and Rolling Torque. The calculation of rolling force can be performed by the following formula:

$$
F_{r}=\sigma_{a} \cdot \bar{\sigma} \cdot l_{d} \cdot b_{\mathrm{av}}
$$

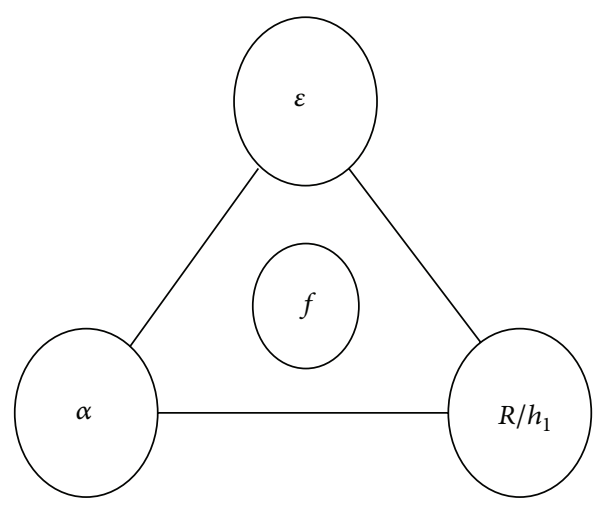

FIGURE 29: Rolling gap parameters.

and rolling torque of two rolls can be performed by the following formula:

$$
M_{r}=2 F_{r} \cdot a
$$

The arm of rolling force resulting from (122) can be expressed as follows:

$$
a=\psi \cdot l_{d}
$$

Substituting (129) and (131) into (130) formula for calculation of rolling torque is obtained:

$$
M_{r}=2 R \cdot \sigma_{a} \cdot \bar{\sigma} \cdot \psi \cdot \Delta h \cdot b_{\mathrm{av}} .
$$

The value of coefficient of arm of rolling force $\psi$ is given in Figure 28.

\section{Discussion}

The solution of difference equation (1) realized by the authors of [15-18] was based on determining only one constant $m$ which consists of product of friction coefficient and geometrical characteristics of rolling gap.

The constant according to Korolev [15] $m_{\text {Kor }}$ described by (13) is depending on product of friction coefficient and ratio $l_{d} / h_{\mathrm{av}}$. The constant according to Tselikov et al. [16] $m_{\text {Tsel }}$ described by (15) is depending on product of friction coefficient and ratio $2 l_{d} /\left(h_{0}-h_{1}\right)$. The constant according to Bland-Ford [17] $m_{\text {Bla-For }}$ described by (22) is depending on product of friction coefficient and ratio $\sqrt{R / h_{1}}$. The constant according to Smiryagin-Pernisov [19] $m_{\text {Smir-Per }}$, described by (30), is depending on product of friction coefficient and ratio $2 l_{d} / \sqrt{h_{1}\left(h_{0}-h_{1}\right)}$.

In the new solution of difference equation (1) presented by this paper from calculation of $m$ constant is separating friction coefficient and is described by (51) which is depending only on geometry of rolling gap given by ratio $R / h_{1}$.

Each of constants is described by (13), (15), and (22). Equation (30) contains deformation in some form, but the deformation does not directly include (74) and (75). The interrelationship characteristics of rolling gap can be schematically shown in Figure 29. 
TABLE 5: The calculations $\bar{\sigma}$ function according to (95) and $\bar{\sigma}_{\text {Kor }}$ function by a Korolev.

\begin{tabular}{|c|c|c|c|c|c|c|c|c|c|c|}
\hline $\begin{array}{l}h_{0} \\
(\mathrm{~mm})\end{array}$ & $\begin{array}{c}h_{1} \\
(\mathrm{~mm})\end{array}$ & $\begin{array}{c}\varepsilon \\
(-)\end{array}$ & $\begin{array}{c}R \\
(\mathrm{~mm})\end{array}$ & $\begin{array}{c}R / h_{1} \\
(-)\end{array}$ & $\begin{array}{c}l_{d} / h_{\mathrm{av}} \\
(-)\end{array}$ & $\begin{array}{c}f \\
(-)\end{array}$ & $\begin{array}{c}m_{\text {Kor }} \\
(-)\end{array}$ & $\begin{array}{c}\bar{\sigma}_{\text {Kor }} \\
(-)\end{array}$ & $\begin{array}{c}\bar{\sigma} \\
(-)\end{array}$ & $\begin{array}{c}\bar{\sigma}_{\text {Kor }} / \bar{\sigma} \\
(-)\end{array}$ \\
\hline 2,0 & 1,5259 & 0,2371 & 105 & 68,813 & 4,000 & 0,1 & 0,400 & 1,2296 & 1,2742 & 0,9650 \\
\hline 5,0 & 4,1935 & 0,1613 & 105 & 25,038 & 2,000 & 0,2 & 0,400 & 1,2296 & 1,2586 & 0,9770 \\
\hline 7,0 & 6,2550 & 0,1064 & 105 & 16,786 & 1,3334 & 0,3 & 0,400 & 1,2296 & 1,2482 & 0,9851 \\
\hline
\end{tabular}

TABLE 6: The calculations $\bar{\sigma}$ function according to (95) and $\bar{\sigma}_{\text {Tsel }}$ function by Tselikov.

\begin{tabular}{lcccccccrrr}
\hline $\begin{array}{l}h_{0} \\
(\mathrm{~mm})\end{array}$ & $\begin{array}{c}h_{1} \\
(\mathrm{~mm})\end{array}$ & $\begin{array}{c}\varepsilon \\
(-)\end{array}$ & $\begin{array}{c}R \\
(\mathrm{~mm})\end{array}$ & $\begin{array}{c}R / h_{1} \\
(-)\end{array}$ & $\begin{array}{c}l_{d} / h_{\text {av }} \\
(-)\end{array}$ & $\begin{array}{c}f \\
(-)\end{array}$ & $\begin{array}{c}m_{\text {Tsel }} \\
(-)\end{array}$ & $\begin{array}{c}\bar{\sigma}_{\text {Tsel }} \\
(-)\end{array}$ & $\begin{array}{c}\bar{\sigma} \\
(-)\end{array}$ & $\begin{array}{c}\bar{\sigma}_{\text {Tsel }} / \bar{\sigma} \\
(-)\end{array}$ \\
\hline 2,0 & 1,5338 & 0,2331 & 105 & 68,455 & 3,957 & 0,1 & 3,000 & 1,2024 & 1,2705 & 0,9463 \\
5,0 & 3,1416 & 0,3717 & 105 & 33,422 & 3,424 & 0,2 & 3,000 & 1,3930 & 1,5299 & 0,9106 \\
7,0 & 2,8416 & 0,5941 & 105 & 36,951 & 4,225 & 0,3 & 3,000 & 1,9655 & 2,5319 & 0,7763 \\
\hline
\end{tabular}

TABLE 7: The calculations $\bar{\sigma}$ function according to (95) and $\bar{\sigma}_{\text {Bla-For }}$ function by Bland-Ford.

\begin{tabular}{|c|c|c|c|c|c|c|c|c|c|c|}
\hline $\begin{array}{l}h_{0} \\
(\mathrm{~mm})\end{array}$ & $\begin{array}{c}h_{1} \\
(\mathrm{~mm})\end{array}$ & $\begin{array}{c}\varepsilon \\
(-)\end{array}$ & $\begin{array}{c}R \\
(\mathrm{~mm})\end{array}$ & $\begin{array}{l}R / h_{1} \\
(-)\end{array}$ & $\begin{array}{c}l_{d} / h_{\mathrm{av}} \\
(-)\end{array}$ & $\begin{array}{c}f \\
(-)\end{array}$ & $\begin{array}{c}m_{\text {Bla-For }} \\
(-)\end{array}$ & $\begin{array}{c}\bar{\sigma}_{\text {Bla-For }} \\
(-)\end{array}$ & $\begin{array}{c}\bar{\sigma} \\
(-)\end{array}$ & $\begin{array}{c}\bar{\sigma}_{\text {Bla-For }} / \bar{\sigma} \\
(-)\end{array}$ \\
\hline 0,328 & 0,2625 & 0,2 & 105 & 400 & 8,888 & 0,1 & 2,000 & 1,5573 & 1,7177 & 0,9066 \\
\hline 1,500 & 1,0500 & 0,3 & 105 & 100 & 5,388 & 0,2 & 2,000 & 1,7075 & 2,0049 & 0,8517 \\
\hline 3,938 & 2,3625 & 0,4 & 105 & 44,444 & 4,075 & 0,3 & 2,000 & 1,8295 & 2,3162 & 0,7897 \\
\hline
\end{tabular}

TABLE 8: The calculations $\bar{\sigma}$ function according to (95) and $\bar{\sigma}_{\text {Sim }}$ function by Sims.

\begin{tabular}{|c|c|c|c|c|c|c|c|c|c|c|}
\hline $\begin{array}{l}h_{0} \\
(\mathrm{~mm})\end{array}$ & $\begin{array}{c}h_{1} \\
(\mathrm{~mm})\end{array}$ & $\begin{array}{c}\varepsilon \\
(-)\end{array}$ & $\begin{array}{c}R \\
(\mathrm{~mm})\end{array}$ & $\begin{array}{c}R / h_{1} \\
(-)\end{array}$ & $\begin{array}{c}l_{d} / h_{\mathrm{av}} \\
(-)\end{array}$ & $\begin{array}{c}f \\
(-)\end{array}$ & $\begin{array}{c}m_{\text {Sim }} \\
(-)\end{array}$ & $\begin{array}{l}\bar{\sigma}_{\text {Sim }} \\
(-)\end{array}$ & $\begin{array}{c}\bar{\sigma} \\
(-)\end{array}$ & $\begin{array}{c}\bar{\sigma}_{\text {Sim }} / \bar{\sigma} \\
(-)\end{array}$ \\
\hline 6,0 & 4,2 & 0,3 & 105 & 25 & 2,690 & 0,1 & 5,000 & 1,4380 & 1,2493 & 1,1510 \\
\hline 6,0 & 4,2 & 0,3 & 105 & 25 & 2,690 & 0,2 & 5,000 & 1,4380 & 1,4540 & 0,9890 \\
\hline 6,0 & 4,2 & 0,3 & 105 & 25 & 2,690 & 0,3 & 5,000 & 1,4380 & 1,7011 & 0,8453 \\
\hline
\end{tabular}

From Figure 29 and the Appendix the result is the possibility of mutual replacement of one parameter by the other, but friction coefficient does not depend on any of these parameters. The new solution of differential equation (1) based on calculation of the relative normal contact stress $\bar{\sigma}$ depending on two functions can be compared with calculation of $\bar{\sigma}$ functions according to the authors of [1518]. The examples of calculations of $\bar{\sigma}$ functions according to the authors of [15-18] with comparison of calculation $\bar{\sigma}$ function according to (95) are given in Tables $5,6,7$, and 8 . Graphical comparison of calculations of differential equation (1) resulting from various approaches defined by the authors of [15-17] and calculation given by (95) is shown in Figure 30.

The length of rolling gap is defined by relative coordinate $x / l_{d}$. Entry side to rolling gap is marked by point $\mathbf{B}$ and exit side from rolling gap by point $\mathbf{A}$. The maximal values of the relative normal contact stress $\bar{\sigma}$ were obtained by using (95).

\section{Conclusion}

The solutions of von Karman two-dimensional differential equation of lengthwise rolling process were based on certain simplifications which are describing the geometry of surface of circular cylinder. Circular arch of roll gap was so

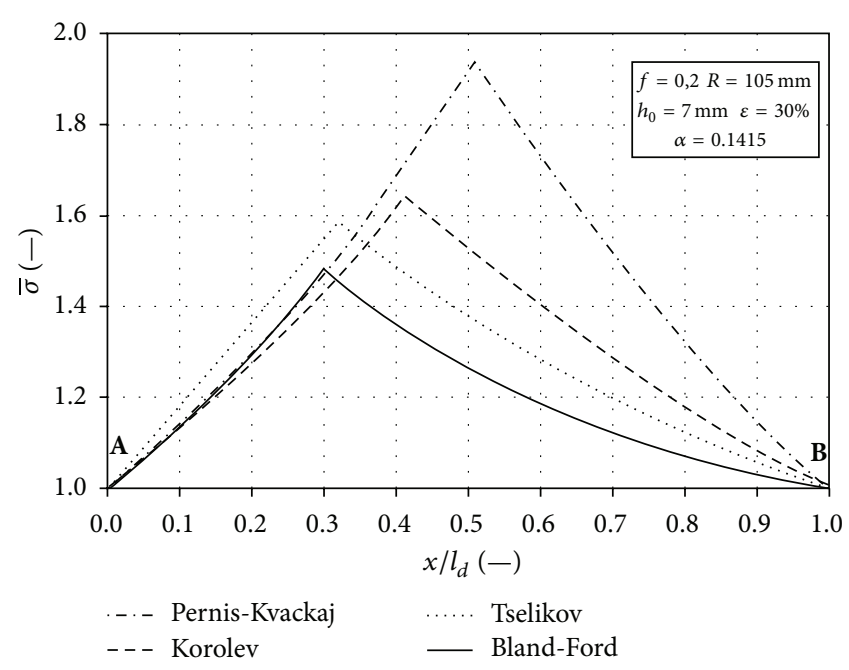

FIGURE 30: The distribution of the relative normal contact stress in rolling gap.

far approximated by simplified curves (polygonal curve or parabola) because his accurate description using the equation circle did not create an analytical solution of the differential 
equation (1). Use of any approximated formulas leads to inaccuracy of calculations relative to normal contact stress along the length of the contact arc. Attempts have been made to describe the contact arc of a circle by the equation of the circle but the exact solution of (1) has been replaced by approximate solution using numerical simulation in software Deform.

The proposed solution presents a new approach to the description of the circular arch of rolling gap by equation of a circle. For this approach which is describing the circular arch of rolling gap by equation of a circle the classical analytical solution of deferential equation cannot be used. Therefore new solution of deferential equation based on interaction two methods was suggested and realized. The authors named this solution with term analytic numerical solution. Also the appropriate solution was the transformation of the coordinate system from rectangular to polar coordinates. The distribution of the relative normal contact stress to rolls is dependent on two independent functions: $\bar{\sigma}_{n} \approx F_{1} \cdot F_{2}$. The first function has exponential form $F_{1}=e^{F(f, \varphi)}$ and is describing the influence of the friction between roll and rolling material. The second function has trigonometric form $F_{2}=F_{2}(m, \varphi)$ and is describing geometry of rolling gap and is independent on friction coefficient.

The results from the numerical calculations are carried out geometric visualizations for neutral section, normal contact stress distribution, front and back tensions, coefficient of the arm of rolling force, rolling force, and rolling torque in depend on rolling conditions.

Result from laboratory cold rolled experiment of $\mathrm{CuZn} 30$ material was derived equation describing work hardening of observed material. This equation was used for calculation of the relative normal contact stress $\bar{\sigma}$ with consideration of work hardening during material processing. Comparison of theoretical values of $\bar{\sigma}$ function calculated according to (95) with values of $\bar{\sigma}$ function calculated based on experimental results from cold rolled was performed. The solution of (1) was not concluded with roll flattening.

\section{Appendix}

Absolute deformation is as follows:

$$
\Delta h=h_{0}-h_{1} .
$$

Relative deformation is as follows:

$$
\varepsilon=\frac{h_{0}-h_{1}}{h_{0}} .
$$

True strain is as follows:

$$
\bar{\varepsilon}=\ln \left(\frac{h_{0}}{h_{1}}\right),
$$

respectively,

$$
\bar{\varepsilon}=-\ln (1-\varepsilon) .
$$

Ratio $h_{1} / h_{0}: \rightarrow h_{1} / h_{0}=1-\varepsilon$.
Average thickness is as follows:

$$
h_{\mathrm{av}}=\frac{h_{0}+h_{1}}{2} .
$$

Gripping angle is as follows:

$$
\cos \alpha=1-\frac{\Delta h}{2 R}
$$

respectively,

$$
\cos \alpha=1-\frac{\varepsilon}{1-\varepsilon} \cdot \frac{h_{1}}{2 R} .
$$

Length of contact arc is as follows:

$$
l_{d}=\sqrt{R \Delta h-\frac{\Delta h^{2}}{4}}
$$

respectively,

$$
l_{d} \doteq \sqrt{R \Delta h}
$$

Ratio $l_{d} / h_{\mathrm{av}}: \rightarrow l_{d} / h_{\mathrm{av}}=2\left(\sqrt{R \Delta h} /\left(h_{0}+h_{1}\right)\right)$.

Ratio $R / h_{1}: \rightarrow R / h_{1}=(2-\varepsilon)^{2} / 4 \varepsilon(1-\varepsilon) \cdot\left(l_{d} / h_{\mathrm{av}}\right)^{2}$, respectively, $R / h_{1}=\varepsilon / 2(1-\varepsilon) \cdot 1 /(1-\cos \alpha)$.

Relative deformation is as follows:

$$
\varepsilon=\frac{1}{1+h_{1} / 2 R \cdot 1 /(1-\cos \alpha)} .
$$

\section{Nomenclature}

a: $\quad$ Arm force $[\mathrm{m}]$

$b_{\mathrm{av}}: \quad$ Average width $[\mathrm{m}]$

$C_{B}$ : Integration constant (Backward slip zone) $[-]$

$C_{F}$ : Integration constant (Forward slip zone) [-]

$d x, d y$ : Coordinate differentials $x$ and $y[-]$

$f: \quad$ Friction coefficient [-]

$F_{r}$ : $\quad$ Rolling force [N]

$h_{\mathrm{av}}: \quad$ Average thickness [m]

$h_{n}$ : Thickness in neutral section [m]

$h_{0}, h_{1}$ : Thickness before and after deformation [m]

$l_{d}: \quad$ Length of contact arc [m]

$m$ : Constant of differential equation [-]

$M_{r}: \quad$ Rolling torque [Nm]

$R: \quad$ Radius of rollers [m]

$x, y: \quad$ Rectangular coordinates $[\mathrm{m}]$

YS: Uniaxial yield stress [MPa]

$\alpha$ : $\quad$ Gripping angle [rad] 


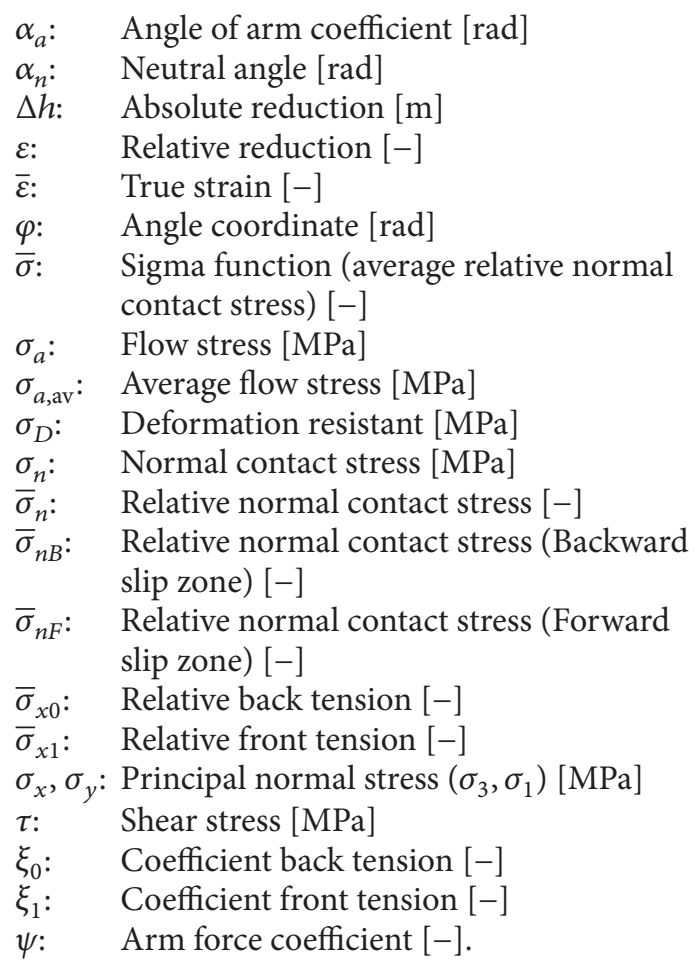

\section{Conflict of Interests}

The authors declare that there is no conflict of interests regarding the publication of this paper.

\section{Acknowledgments}

This work was financially supported by the VEGA 1/0325/14 project and the project "Technological Preparation of Electrotechnical Steels with High Permeability for Electrodrives with Higher Efficiency" which is supported by the Operational Program "Research and Development" ITMS 26220220037, financed through European Regional Development Fund.

\section{References}

[1] T. Karman, "Beitrag zur Theorie des Walzvorganges," Zeitschrift für Angewandte Mathematik und Mechanik, vol. 5, no. 2, pp. 139-141, 1925 (German).

[2] B. Avitzur, Metal Forming: Processes and Analysis, McGraw-Hill, New York, NY, USA, 1968.

[3] A. Hensel and T. Spittel, The Force and Energy Consumption in Metal Forming Processes, Deutscher Verlag für Grundstoffindustrie, Leipzig, Germany, 1978, (German).

[4] E. M. Mielnik, Metalworking Science and Engineering, McGrawHill, New York, NY, USA, 1991.

[5] M. Hajduk and J. Konvičný, Power Requirements for Hot Rolled Steel, SNTL, Prague, Czech Republic, 1983, (Czech).

[6] M. Kollerová, M. Žídek, B. Počta, and V. Dědek, The Rolling, ALFA, Bratislava, Slovakia, 1991 (Slovak).

[7] T. Kubina, J. Bořuta, and I. Schindler, "New possibilities of evaluation of the degree of softening from torsion plastometric test," Acta Metallurgica Slovaca, vol. 13, no. 4, pp. 610-617, 2007.
[8] R. Pernis, J. Kasala, and J. Bořuta, "High temperature plastic deformation of CuZn30 brass-calculation of the activation energy," Kovové Materiály-Metallic Materials, vol. 48, no. 1, pp. 41-46, 2010.

[9] T. Kvackaj, I. Pokorný, and M. Vlado, "Simulácie procesov tvárnenia pomocou krutovej plastometrie," Acta Metallurgica Slovaca, vol. 6, no. 3, pp. 242-248, 2000.

[10] E. Orowan, "The calculation of roll pressure in hot and cold flat rolling," Proceedings of the Institution of Mechanical Engineers, vol. 150, no. 1, pp. 140-167, 1943.

[11] HITACHI Analog, Analysis of Rolling Theory by Analog Computer, Karman's Differential Equation, Hybrid Computer, Technical Information Series No. 8, Hitaschi, 1968.

[12] O. Harrer and I. Pokorný, “The course of stresses and velocities in billet rolling," Acta Metallurgica Slovaca, vol. 9, no. 1, pp. 3440, 2003.

[13] S. I. Gubkin, Plastic Deformation of Metals, Metallurgizdat, Moscow, Russia, 1960, (Russian).

[14] R. Kočiško, J. Bacsó, J. Bidulská, A. Kováčová, P. Bella, and M. Molnárová, "Influence of deformation degree on local deformation heterogeneity during thin sheet rolling," Chemicke Listy, vol. 105, no. 17, pp. s822-s823, 2011.

[15] A. A. Korolev, The Machines and Equipments of Rolling of Ferrous and Nonferrous Metallurgy, Metallurgiya, Moscow, Russia, 1976, (Russian).

[16] A. I. Tselikov, G. S. Nikitin, and S. E. Rokotyan, The Theory of Lengthwise Rolling, MIR Publishers, Moscow, Russia, 1981.

[17] D. R. Bland and H. Ford, "The calculation of roll force and torque in cold strip rolling with tension," Proceedings of the Institution of Mechanical Engineers, vol. 159, no. 1, pp. 144-163, 1948.

[18] R. B. Sims, "Calculation of roll force and torque in hot rolling mills," Proceedings of the Institution of Mechanical Engineers, vol. 168, no. 6, pp. 191-200, 1954.

[19] A. P. Smirjagin, N. Z. Dnestrovskij, A. D. Landichov et al., Processing Manual for Non-Ferrous Metals and Alloys, Metallurgizdat, 1961 (Russian).

[20] R. Pernis, "The new hypothesis of normal stress distribution for contact arc in rolling," Acta Metallurgica Slovaca, vol. 17, no. 2, pp. 68-76, 2011.

[21] L. Matejička, "On an open problem posed in the paper 'inequalities of power-exponential functions," Journal of Inequalities in Pure and Applied Mathematics, vol. 9, no. 3, article 75, 5 pages, 2008.

[22] I. S. Gradshteyn and I. M. Ryzhik, Table of Integrals, Series, and Products, Academic Press, London, UK, 7th edition, 2007.

[23] L. Matejíčka, "On approximate solutions of degenerate integrodifferential parabolic problems," Mathematica Slovaca, vol. 45, no. 1, pp. 91-103, 1995.

[24] A. Angot, Complément de Mathématiques à l'usage des Ingénieurs de l'Electrotechnique et des Télécommunications, MASSON, Paris, France, 1957.

[25] J. M. Alexander, "On the theory of rolling," Proceedings of the Royal Society of London A, vol. 326, pp. 535-563, 1972. 


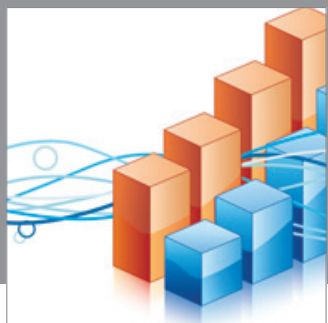

Advances in

Operations Research

mansans

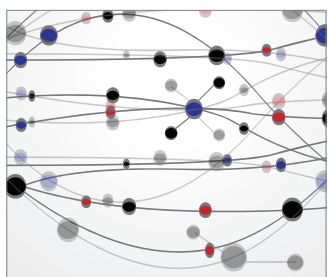

The Scientific World Journal
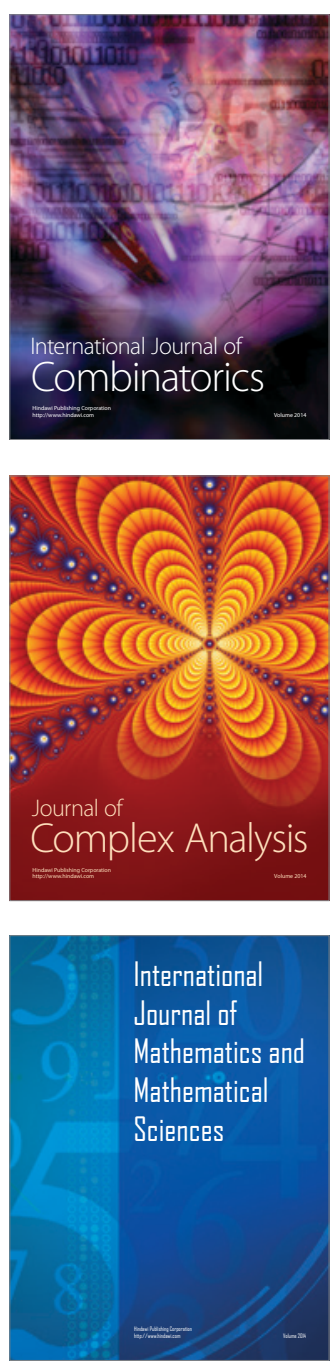
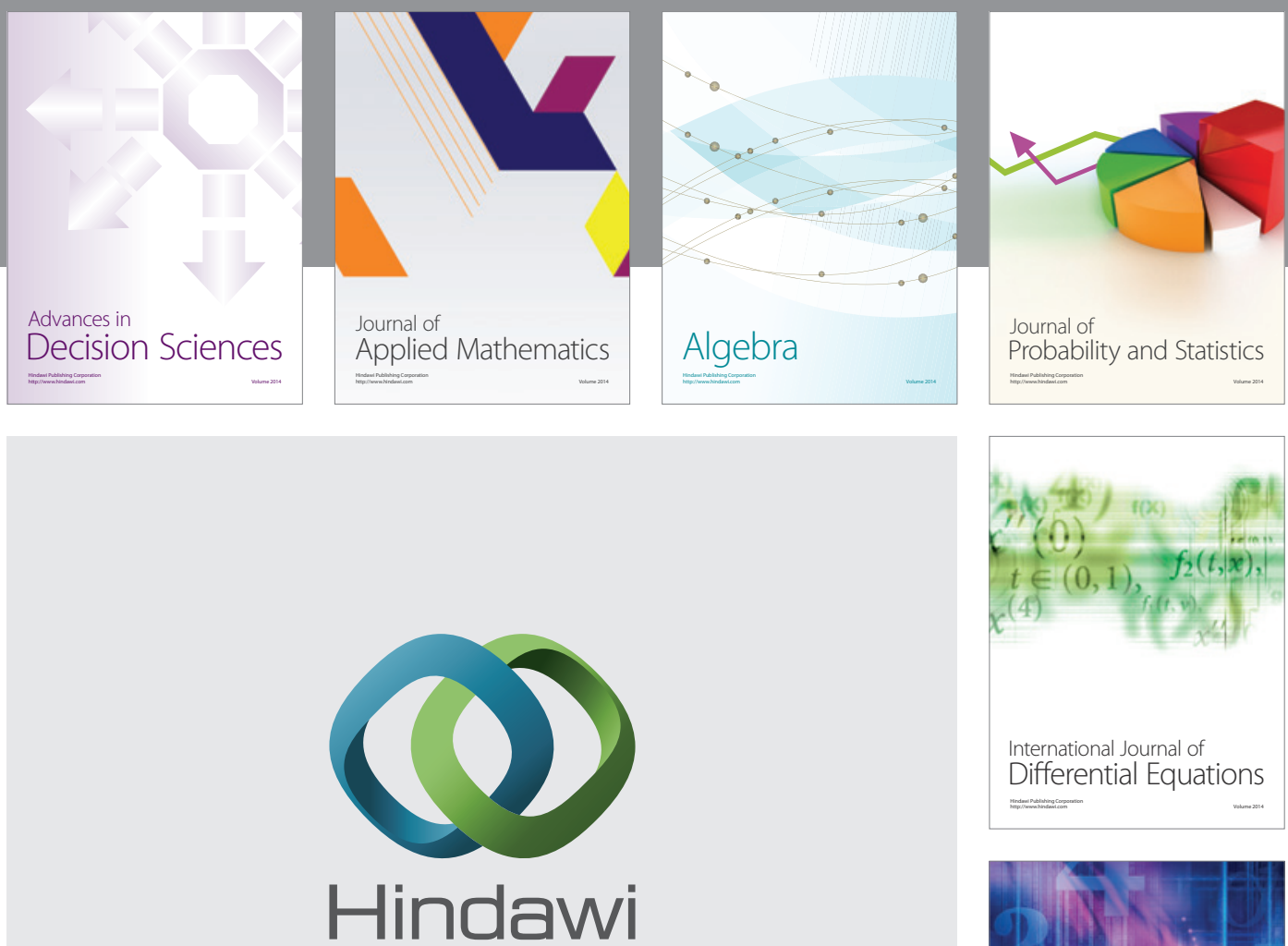

Submit your manuscripts at http://www.hindawi.com
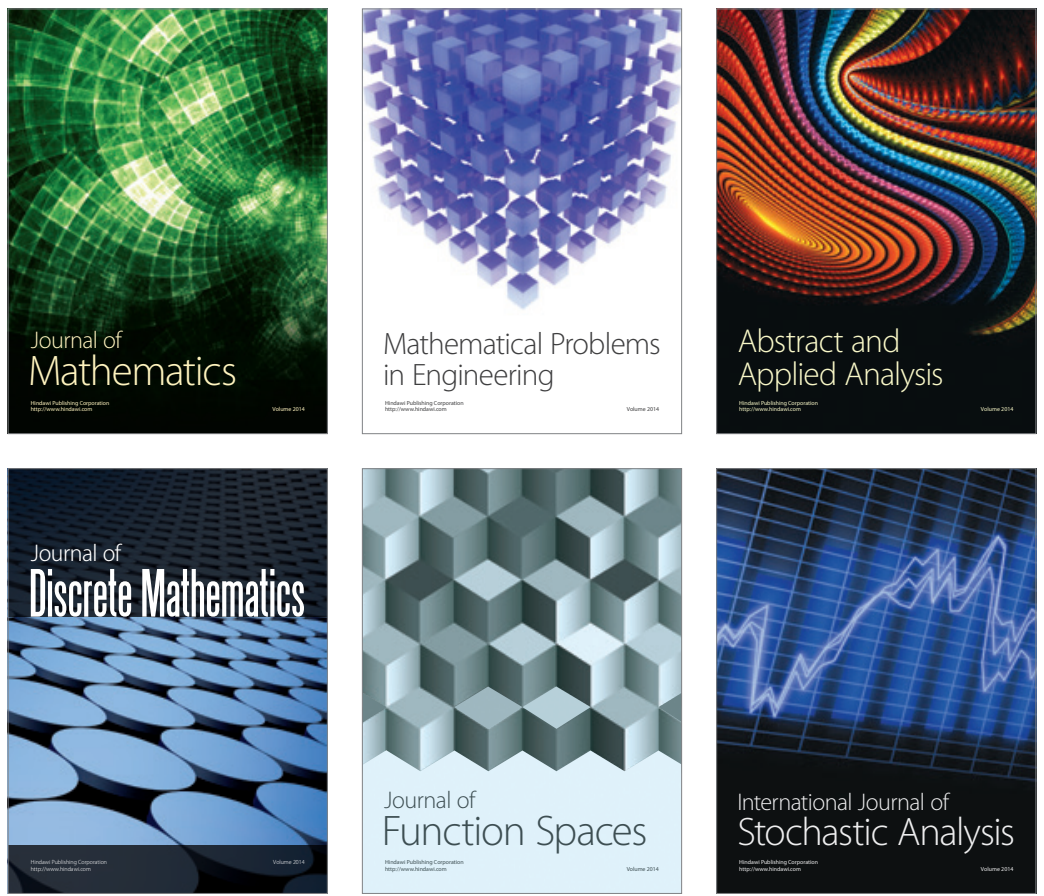

Journal of

Function Spaces

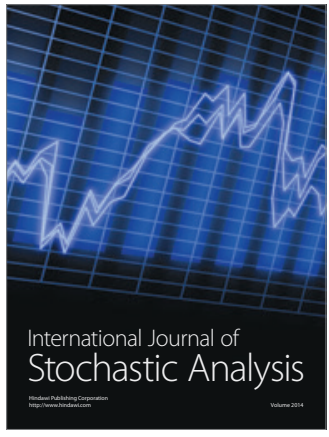

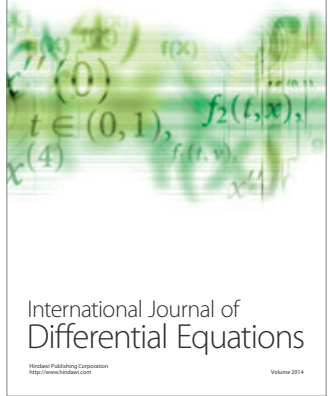
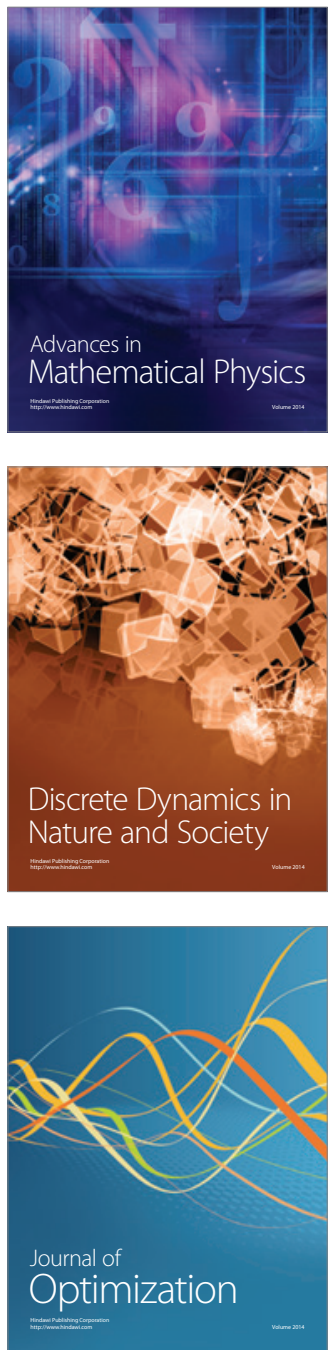